\title{
Construction Warehouse
}

Culminating Experience in Fire Protection Engineering

FPE 596

Prepared by

Ben Johnson

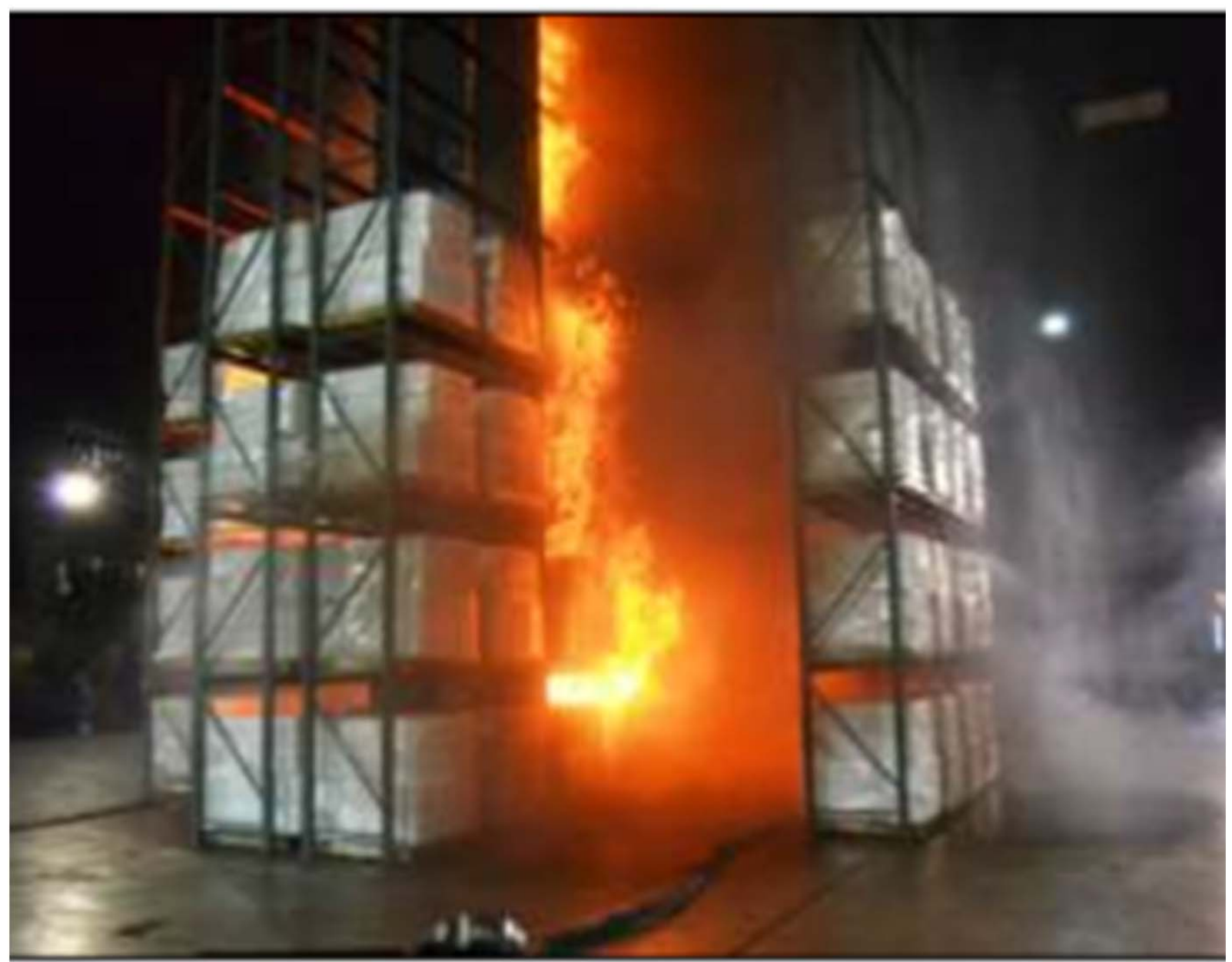

Cal Poly

Fire Protection Engineering 


\section{Construction Warehouse}

Waste Treatment and Immobilization Plant (WTP) Project

- U.S. Department of Energy (DOE) Project on the Hanford Site in SouthCentral Washington State

- Construction Project

- Non-Reactor Nuclear Facility

- Vitrification of liquid waste

- (i.e., transforming liquid waste into glass)

- Project Information

- http://www.hanfordvitplant.com

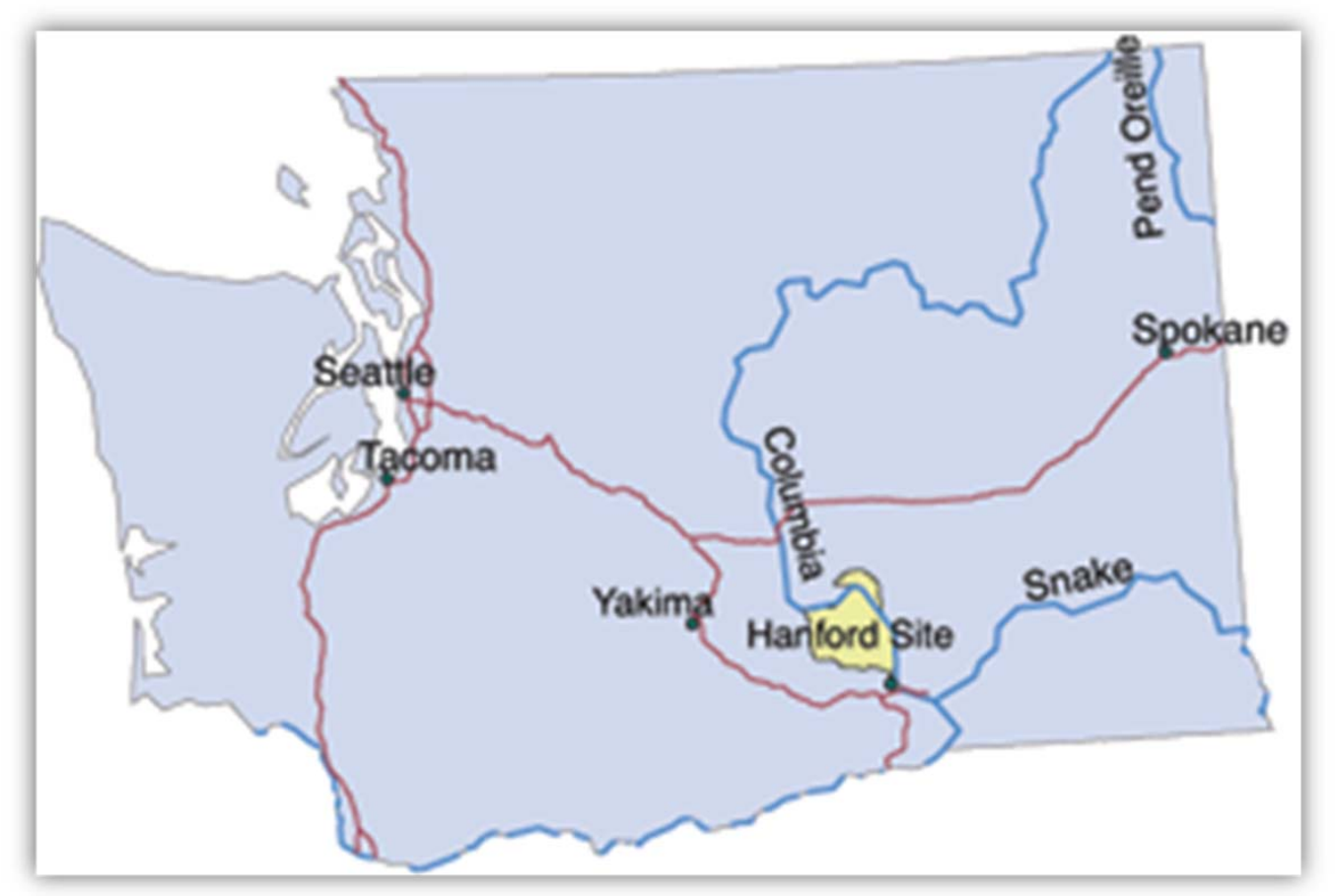

CAL Poly

Fire Protection Engineering 


\section{Construction Warehouse}

Fire Protection Design Criteria

- Original Construction (2002)

- Uniform Building Code (UBC 1997 Edition) - Type II-N

- NFPA 13 - 1999 Edition, Standard for the Installation of Sprinkler Systems

- NFPA 72 - 2002 Edition, National Fire Alarm Code ${ }^{\circledR}$

- NFPA 101 - 1997 Edition, Life Safety Code ${ }^{\circledR}$

- Evaluated to

- International Building Code (IBC 2009 Edition) - Type II-B

- NFPA 13 - 2010 Edition, Standard for the Installation of Sprinkler Systems

- NFPA 72 - 2010 Edition, National Fire Alarm and Signaling Code

- NFPA 101 - 2012 Edition, Life Safety Code ${ }^{\circledR}$ 


\section{Construction Warehouse - Occupancy and Exit Locations Main Floor}

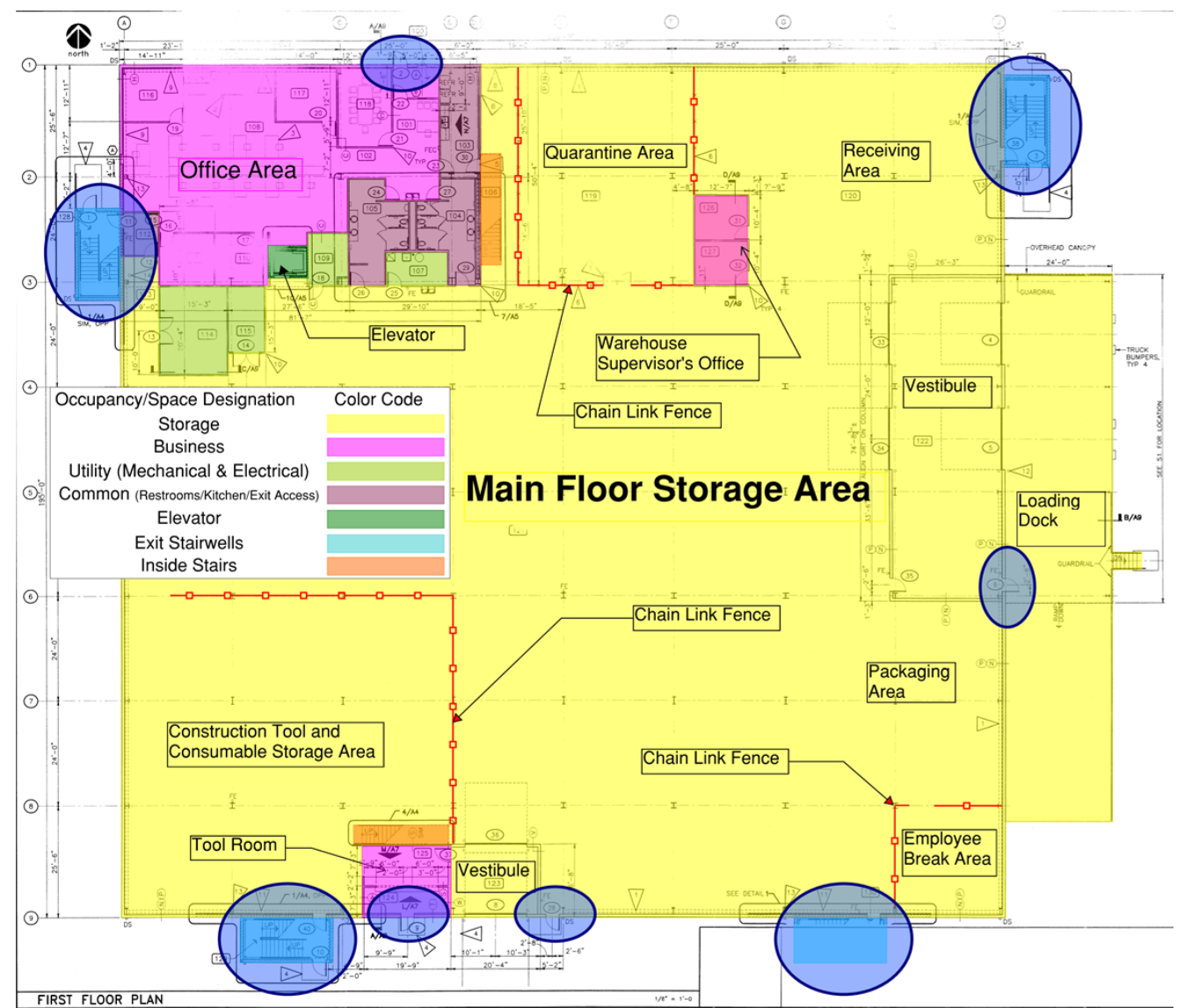

- Main Floor Elevation 0'-0"

- Footprint: $195 \mathrm{ft}$ x $200 \mathrm{ft}$

- Gross Floor Area: $39,000 \mathrm{ft}^{2}$

- Walled-in Office Area:

- Footprint: $50 \mathrm{ft} \times 82 \mathrm{ft}$

- Floor Area: 4,100 $\mathrm{ft}^{2}$

- Occupant Load: 74 persons

- Main Storage Area:

- Floor Area: 34,900 ft²

- Occupant Load: 70 persons 


\section{Construction Warehouse - Occupancy and Exit Locations Mezzanine Level}

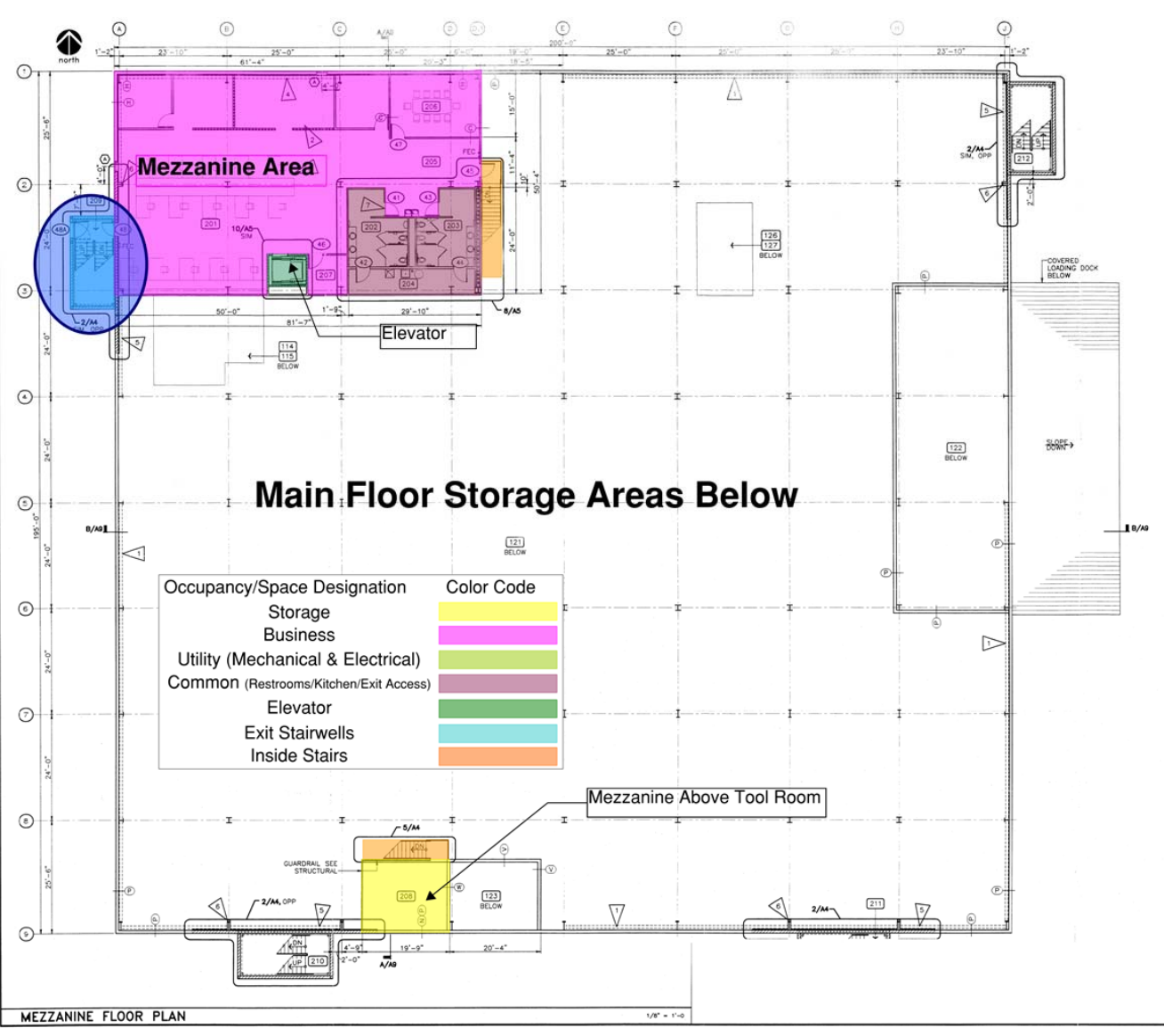

- Mezzanine Elevation 12'-0"

- Walled-in Mezzanine Area:

- Footprint: $50 \mathrm{ft}$ x $82 \mathrm{ft}$

- Floor Area: 4,100 ft ${ }^{2}$

- Occupant Load: 65 persons

- Dispersal Mezzanine Elevation 10'-0"

- Footprint: $15 \mathrm{ft} \times 20 \mathrm{ft}$

- Floor Area: $300 \mathrm{ft}^{2}$

- Occupant Load: 1 person 


\section{Construction Warehouse - Occupancy and Exit Locations Second Floor}

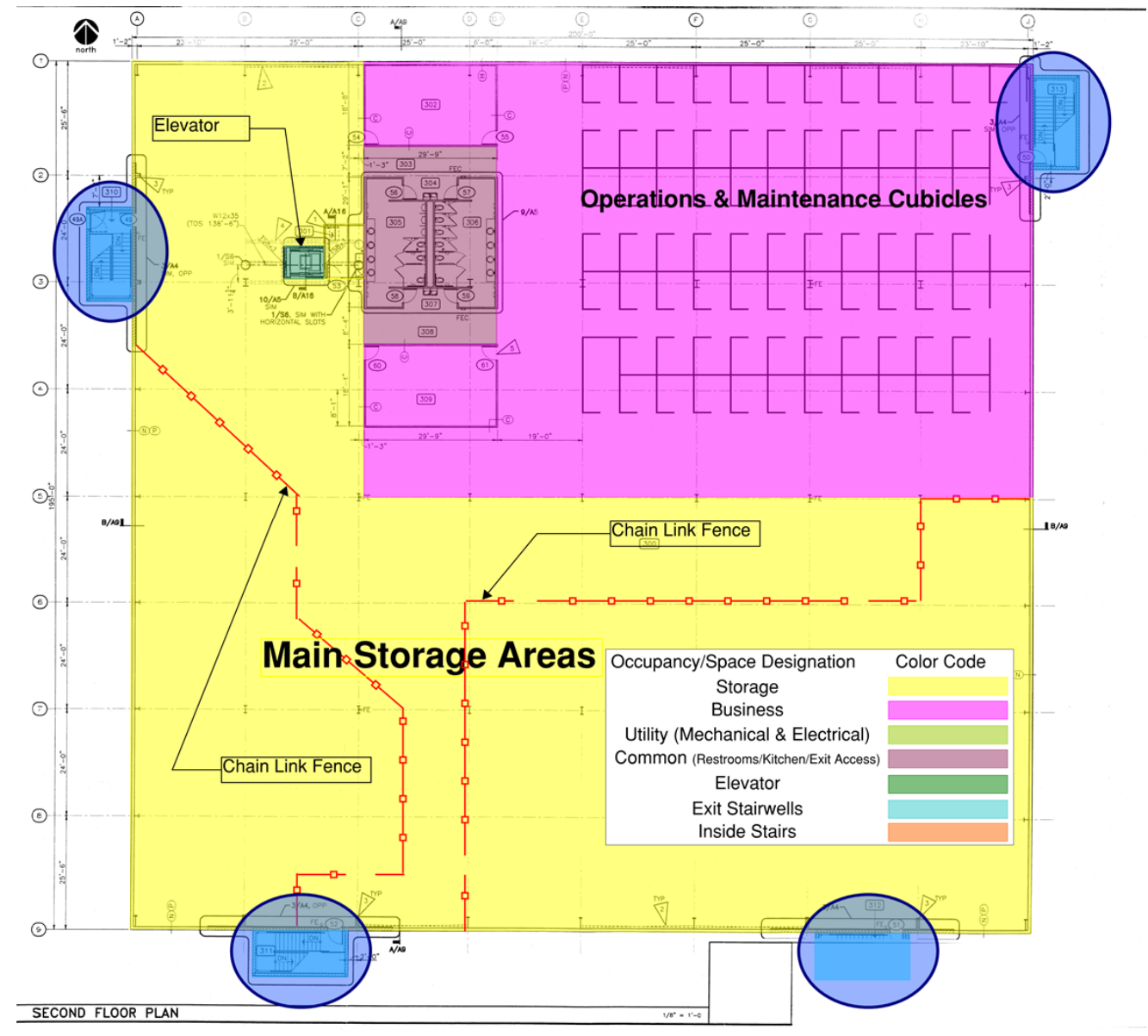

- Second Floor Elevation 24'-0"

- Gross Floor Area: $39,000 \mathrm{ft}^{2}$

- Operations \& Maintenance Offices:

- Footprint: $150 \mathrm{ft} \times 98 \mathrm{ft}$

- Floor Area: $14,700 \mathrm{ft}^{2}$

- Occupant Load: 207 persons

- Second Floor Storage Areas:

- Floor Area: 24,300 ft ${ }^{2}$

- Occupant Load: 51 persons 


\section{Construction Warehouse Building Description}

- Metal Building

- Non-combustible, unprotected, structural steel

- Concrete floors

- Gypsum board on steel stud interior walls

- Pre-finished exterior metal wall panels

- Standing seam metal roof

- Ceiling and wall insulation with exposed vapor barrier facing

- Installed Fire Systems

- Automatic fire sprinkler system

- Fire alarm system 


\section{Construction Warehouse Building Description}

- Evaluate to International Building Code - 2009 Edition

- "Mixed Use" Occupancy

- Storage (S-1) and Business (B)

TABLE 508.4

REQUIRED SEPARATION OF OCCUPANCIES (HOURS)

\begin{tabular}{|c|c|c|c|c|c|c|c|c|c|c|c|c|c|c|c|c|c|c|}
\hline \multirow[b]{2}{*}{ OCCUPANCY } & \multicolumn{2}{|c|}{$A^{d}, E$} & \multicolumn{2}{|c|}{$|-1|-3,, \mid-4$} & \multicolumn{2}{|c|}{$1-2$} & \multicolumn{2}{|c|}{$\mathbf{R}$} & \multicolumn{2}{|c|}{$F-2, S-2^{b}, U$} & \multicolumn{2}{|c|}{ B, F-1, M, S-1 } & \multicolumn{2}{|c|}{$\mathrm{H}-1$} & \multicolumn{2}{|c|}{$\mathrm{H}-2$} & \multicolumn{2}{|c|}{$\mathrm{H}-3, \mathrm{H}-4, \mathrm{H}-5$} \\
\hline & $\mathbf{s}$ & NS & s & NS & $\mathbf{s}$ & NS & $\mathbf{s}$ & NS & s & NS & $\mathbf{s}$ & NS & s & NS & $\mathrm{s}$ & NS & s & NS \\
\hline$A^{d}, E$ & $\mathrm{~N}$ & $\mathrm{~N}$ & 1 & 2 & 2 & NP & 1 & 2 & $\mathrm{~N}$ & 1 & 1 & 2 & NP & $\mathrm{NP}$ & 3 & 4 & 2 & $3^{a}$ \\
\hline I-1, I-3, I-4 & - & - & $\mathrm{N}$ & $\mathrm{N}$ & 2 & $\mathrm{NP}$ & 1 & NP & 1 & 2 & 1 & 2 & NP & NP & 3 & NP & 2 & NP \\
\hline $\mathrm{I}-2$ & - & - & - & - & $\mathrm{N}$ & $\mathrm{N}$ & 2 & NP & 2 & NP & 2 & NP & NP & NP & 3 & NP & 2 & NP \\
\hline $\mathrm{R}$ & - & - & - & - & - & - & $\mathrm{N}$ & $\mathrm{N}$ & $1^{c}$ & $2^{c}$ & 1 & 2 & NP & $\mathrm{NP}$ & 3 & NP & 2 & $\mathrm{NP}$ \\
\hline F-2, S-2 $2^{b}, U$ & - & - & - & - & - & - & - & - & $\mathrm{N}$ & $\mathrm{N}$ & 1 & 2 & $\mathrm{NP}$ & NP & 3 & 4 & 2 & $3^{\mathrm{a}}$ \\
\hline $\begin{array}{c}\text { B, F-1, M, } \\
S-1\end{array}$ & - & - & - & - & - & - & - & - & - & - & $\mathrm{N}$ & $\mathrm{N}$ & NP & NP & 2 & 3 & 1 & $2^{a}$ \\
\hline
\end{tabular}




\section{Construction Warehouse Building Description}

- Evaluate to International Building Code - 2009 Edition

- Two (2) story structure

- Roof Ridge (Height) is $45 \mathrm{ft}$. above grade

- Main Floor (B) Area = 8,200 ft²; (S-1) Area = 34,900 ft²

TABLE 503

ALLOWABLE BUILDING HEIGHTS AND AREAS"

Building height limitations shown in feet above grade plane. Story limitations shown as stories above grade plane. Building area limitations shown in square feet, as determined by the definition of "Area, building," per story

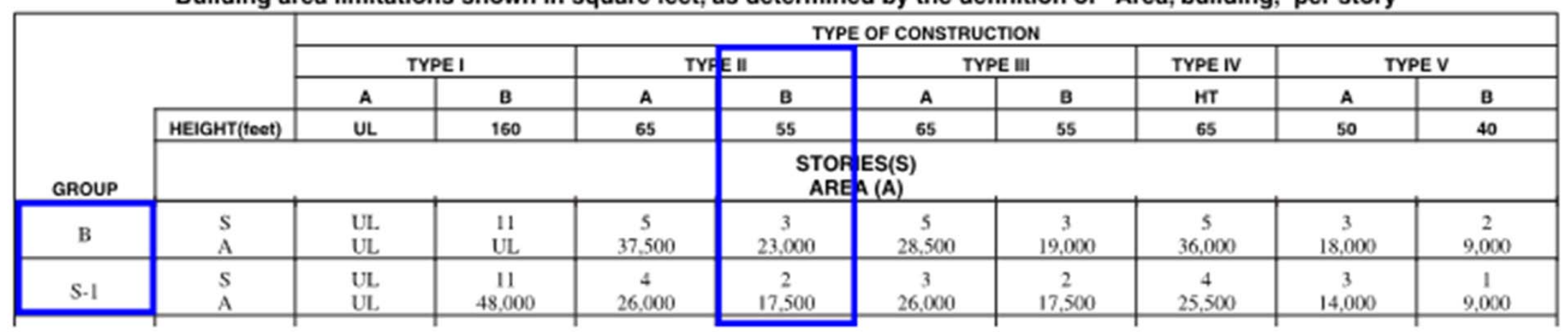




\section{Construction Warehouse Building Description}

- Ratio of Building Area for each Occupancy

- Allowable area modified for frontage and automatic sprinkler system installation

\begin{tabular}{|c|c|c|c|c|c|c|c|}
\hline \multirow[t]{2}{*}{ Floor } & \multicolumn{2}{|c|}{ Occupancy (ft') } & \multicolumn{2}{|c|}{$\begin{array}{l}\text { Allowable Area } \\
\text { for Type II-B }\left(\mathrm{ft}^{2}\right)\end{array}$} & \multicolumn{2}{|c|}{ Ratio } & \multirow{2}{*}{ Sum } \\
\hline & B & S-1 & B & S-1 & B & S-1 & \\
\hline First & \multirow{2}{*}{8,200} & \multirow{2}{*}{34,900} & \multirow{4}{*}{$\begin{array}{r}86,250 \\
(\text { Up from } \\
23,000)\end{array}$} & \multirow{4}{*}{$\begin{array}{r}65,625 \\
\text { (Up from } \\
17,500)\end{array}$} & \multirow{2}{*}{0.10} & \multirow{2}{*}{0.53} & \multirow{2}{*}{0.63} \\
\hline Mezzanine & & & & & & & \\
\hline Second & 24,300 & 14,700 & & & 0.28 & 0.22 & 0.50 \\
\hline Totals & 32,500 & 48,600 & & & & & \\
\hline
\end{tabular}




\section{Construction Warehouse Building Description}

- IBC Type II-B - Fire Resistance Rating of Building Elements

TABLE 601

FIRE-RESISTANCE RATING REQUIREMENTS FOR BUILDING ELEMENTS (hours)

\begin{tabular}{|c|c|c|c|c|c|c|c|c|c|}
\hline \multirow[b]{2}{*}{ BUILDING ELEMENT } & \multicolumn{2}{|c|}{ TYPEI } & \multicolumn{2}{|c|}{ TYPE ॥ } & \multicolumn{2}{|c|}{ TYPE III } & \multirow{2}{*}{$\begin{array}{c}\text { TYPE IV } \\
\text { HT }\end{array}$} & \multicolumn{2}{|c|}{ TYPE V } \\
\hline & A & B & $A^{d}$ & B & $A^{d}$ & B & & $A^{d}$ & B \\
\hline $\begin{array}{l}\text { Primary structural frames } \\
\text { (see Section 202) }\end{array}$ & $3^{\mathrm{a}}$ & $2^{3}$ & 1 & 0 & 1 & 0 & HT & 1 & 0 \\
\hline Bearing walls & & & & & & & & & \\
\hline Exterior $^{\mathrm{f} . \mathrm{z}}$ & 3 & 2 & 1 & 0 & 2 & 2 & 2 & 1 & 0 \\
\hline Interior & $3^{\mathrm{a}}$ & $2^{a}$ & 1 & 0 & 1 & 0 & $1 / \mathrm{HT}$ & 1 & 0 \\
\hline $\begin{array}{l}\text { Nonbearing walls and partitions } \\
\text { Exterior }\end{array}$ & & & & & $\mathrm{Sec}$ & le 6 & & & \\
\hline $\begin{array}{l}\text { Nonbearing walls and partitions } \\
\text { Interior }\end{array}$ & 0 & 0 & 0 & 0 & 0 & 0 & See Section 602.4.6 & 0 & 0 \\
\hline $\begin{array}{l}\text { Floor construction and secondary } \\
\text { members (see Section 202) }\end{array}$ & 2 & 2 & 1 & 0 & 1 & 0 & HT & 1 & 0 \\
\hline $\begin{array}{l}\text { Roof construction and secondary } \\
\text { members (see Section 202) }\end{array}$ & $1^{1 / 1} 2^{b}$ & $1^{\mathrm{b}, \mathrm{c}}$ & $1^{b, c}$ & $0^{\mathrm{e}}$ & $1^{\text {b.e }}$ & 0 & HT & $1^{\text {b.e }}$ & 0 \\
\hline
\end{tabular}




\section{Construction Warehouse Building Description}

- Fire Barriers

- Enclosed Exterior Exit Stairwells (4)

- 2-hour fire rated separation from building interior

- 1-hour fire rated exterior stairwell wall

- 1-hour fire rated separation extended $10 \mathrm{ft}$. on both sides of main building wall

- Elevator Shaft

- 2-hour fire rated separation

- Elevator Machine Room

- 2-hour fire rated separation

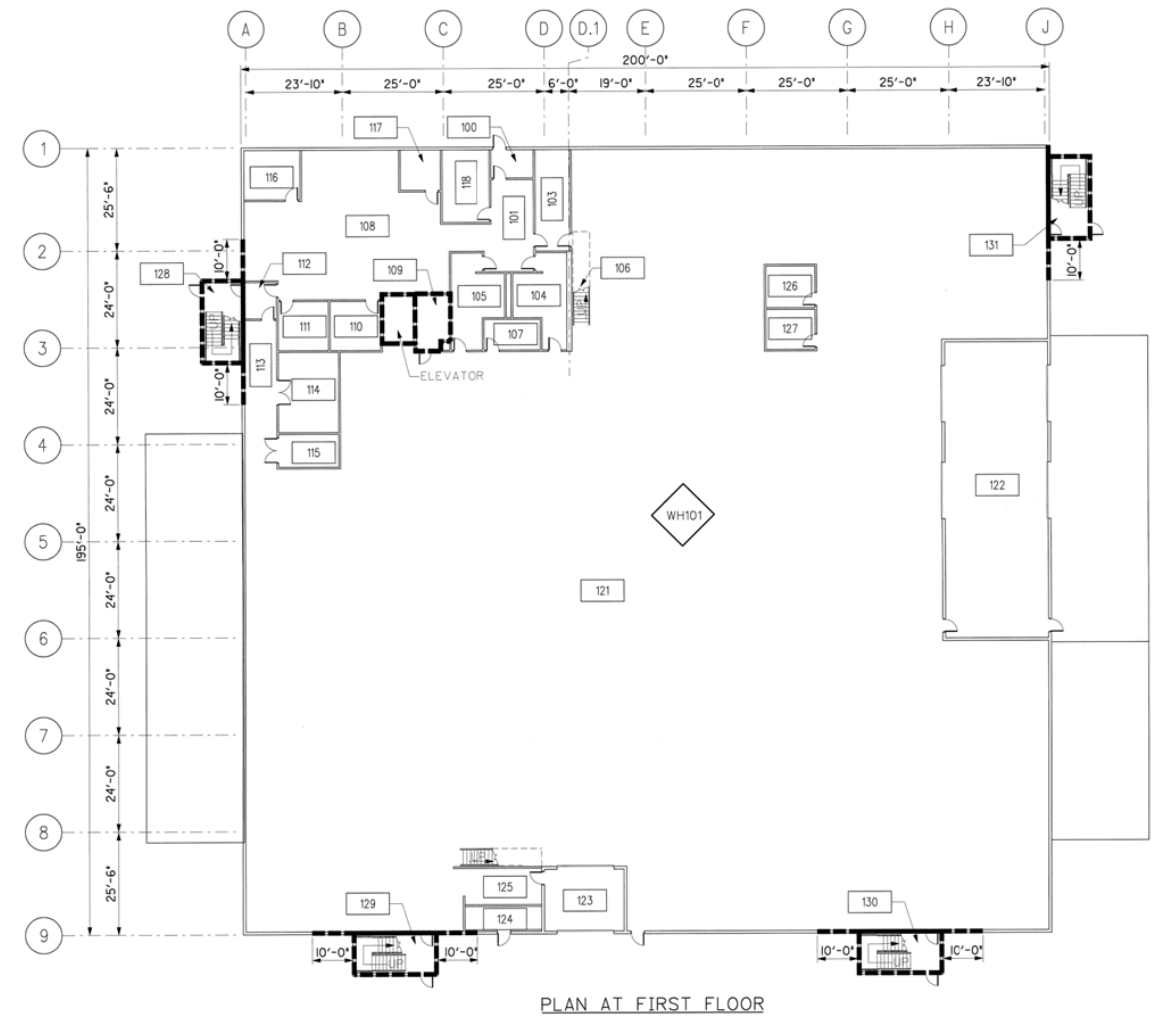

CAL POLY

Fire Protection Engineering 


\section{Construction Warehouse - Water Supply}

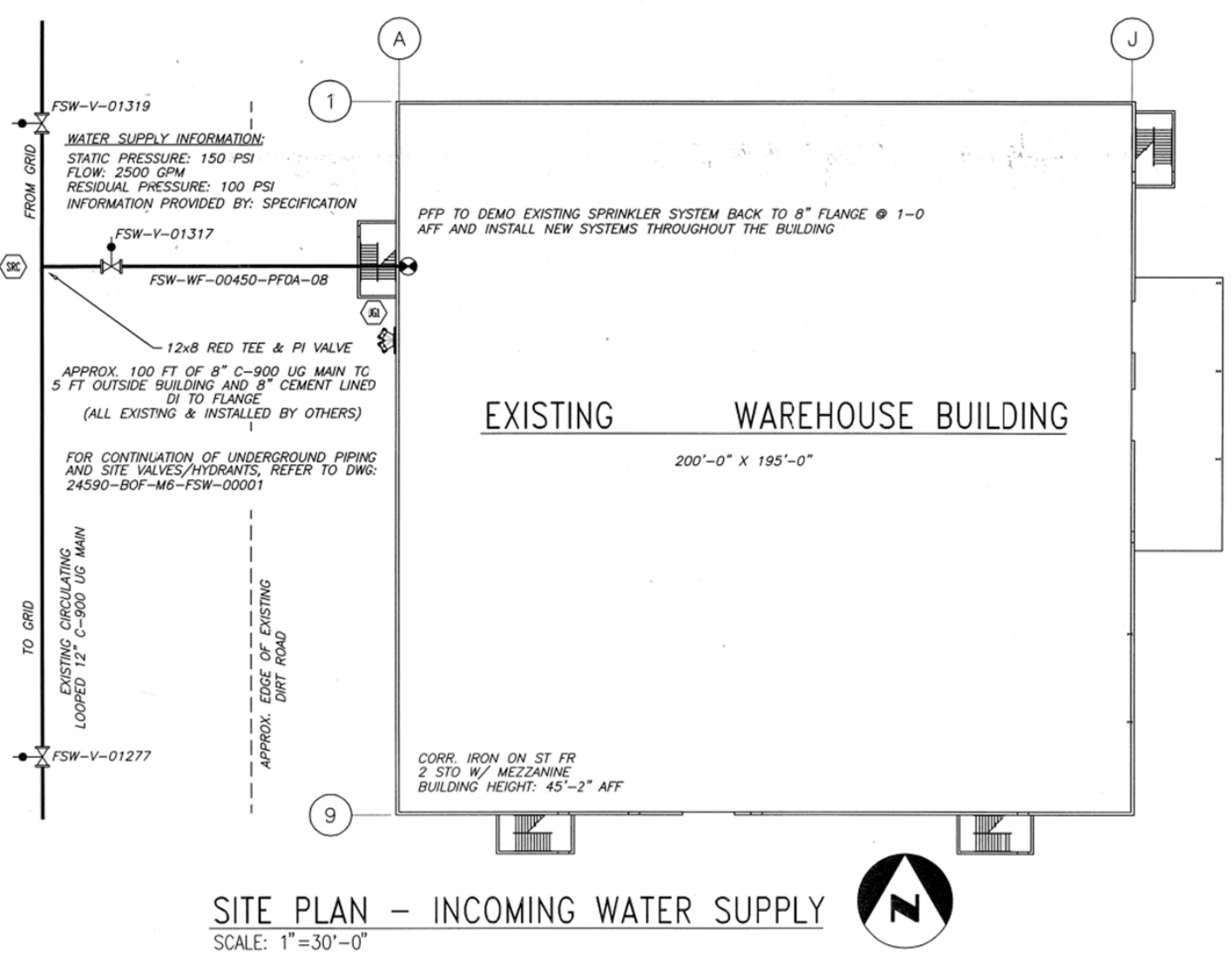

- Two (2) redundant fire water storage tanks

- Each 350,000 gal. capacity

- Two (2) redundant Diesel Fire Pumps

- Each rated at 2,500 gpm @ 130 psi

- 12 inch diameter underground main 


\section{Construction Warehouse - Water Supply}

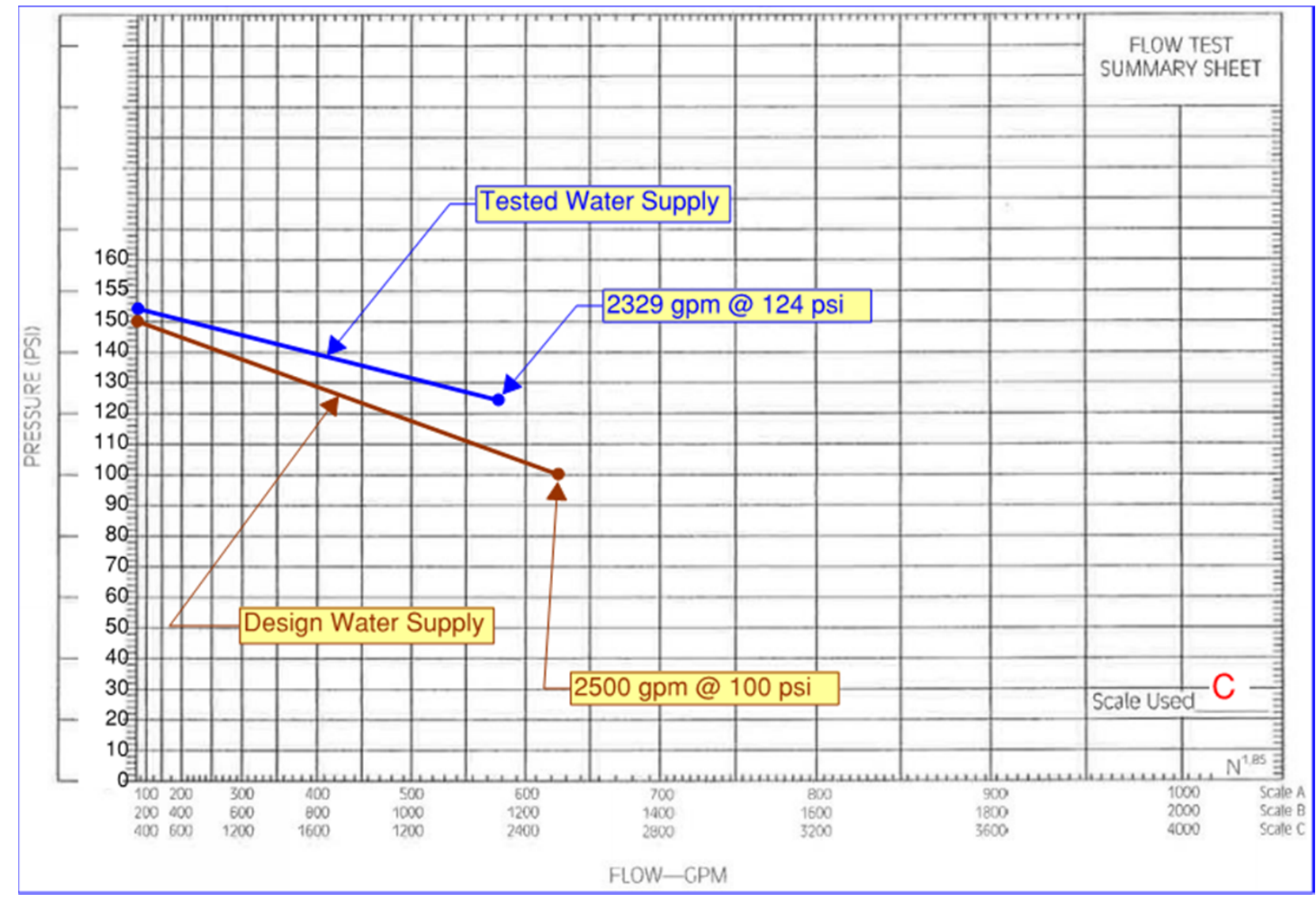

CAL POLY 


\section{Construction Warehouse - Fire Sprinkler System Design Criteria}

\section{Main Floor Storage Area}

- Early Suppression Fast Response (ESFR)

- Group A, unexpanded, exposed, stable plastics

- Single or double-row racks

- Maximum Storage Height of $20 \mathrm{ft}$.

- Minimum $8 \mathrm{ft}$. Aisles

- Gridded system

- ESFR heads, $205^{\circ} \mathrm{F}, \mathrm{K}=14.0$

- Hose stream allowance - $250 \mathrm{gpm}$

- Small hose connections provided for first-aid fire fighting and overhaul operations

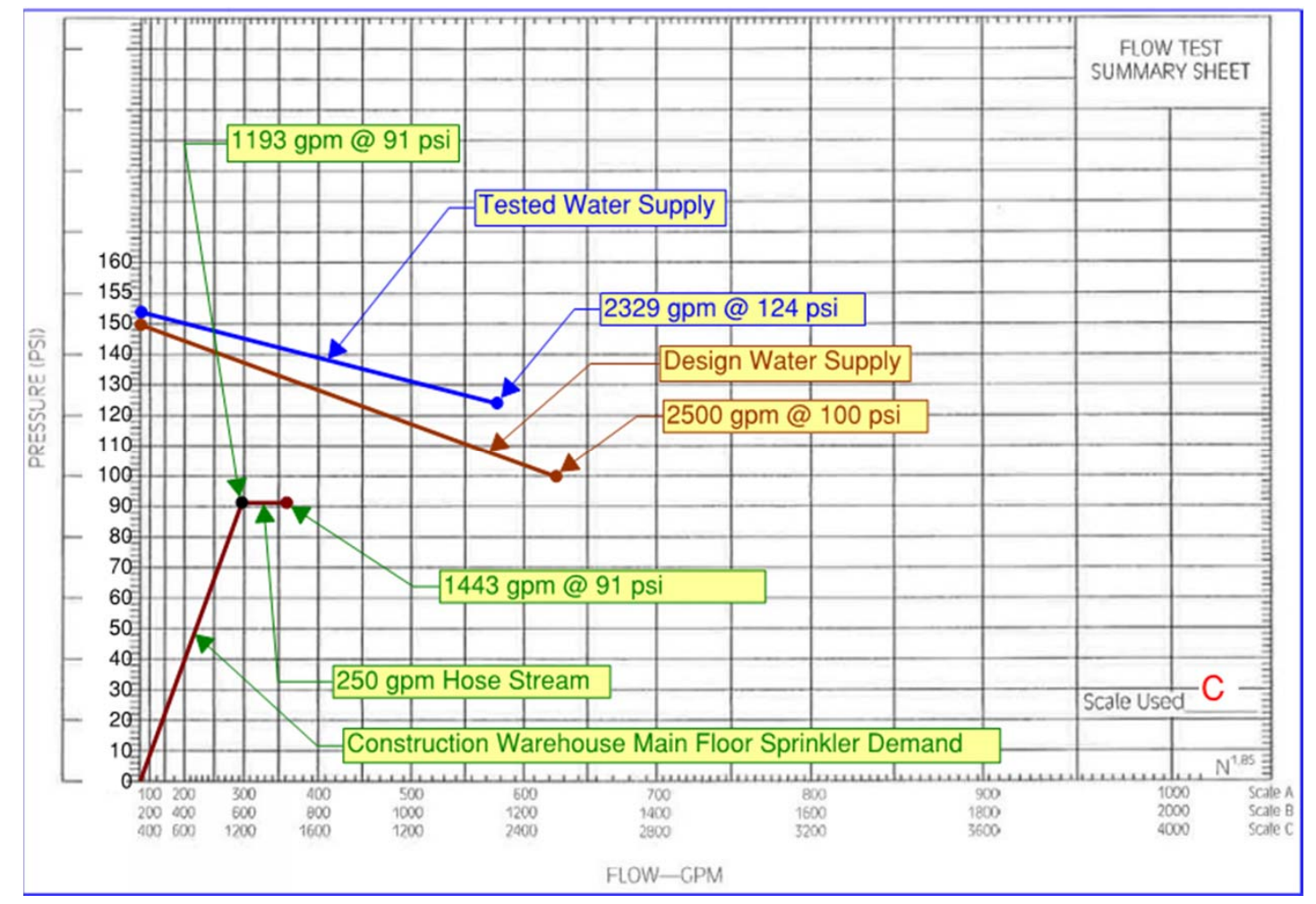




\section{Construction Warehouse - Fire Sprinkler System Design Criteria}

\section{Second Floor}

- Control Mode Density/Area (CMDA)

- Group A, unexpanded, exposed, stable plastics

- Open Shelf or Solid Piled Storage

- Maximum Height of $10 \mathrm{ft}$.

- Gridded system

- Design Area: $0.7 \mathrm{gpm} / 2,500 \mathrm{ft}^{2}$

- Upright Sprinklers, $286^{\circ} \mathrm{F}, \mathrm{K}=16.8$

- Hose stream allowance - $500 \mathrm{gpm}$

- Small hose connections provided for first-aid fire fighting and overhaul operations

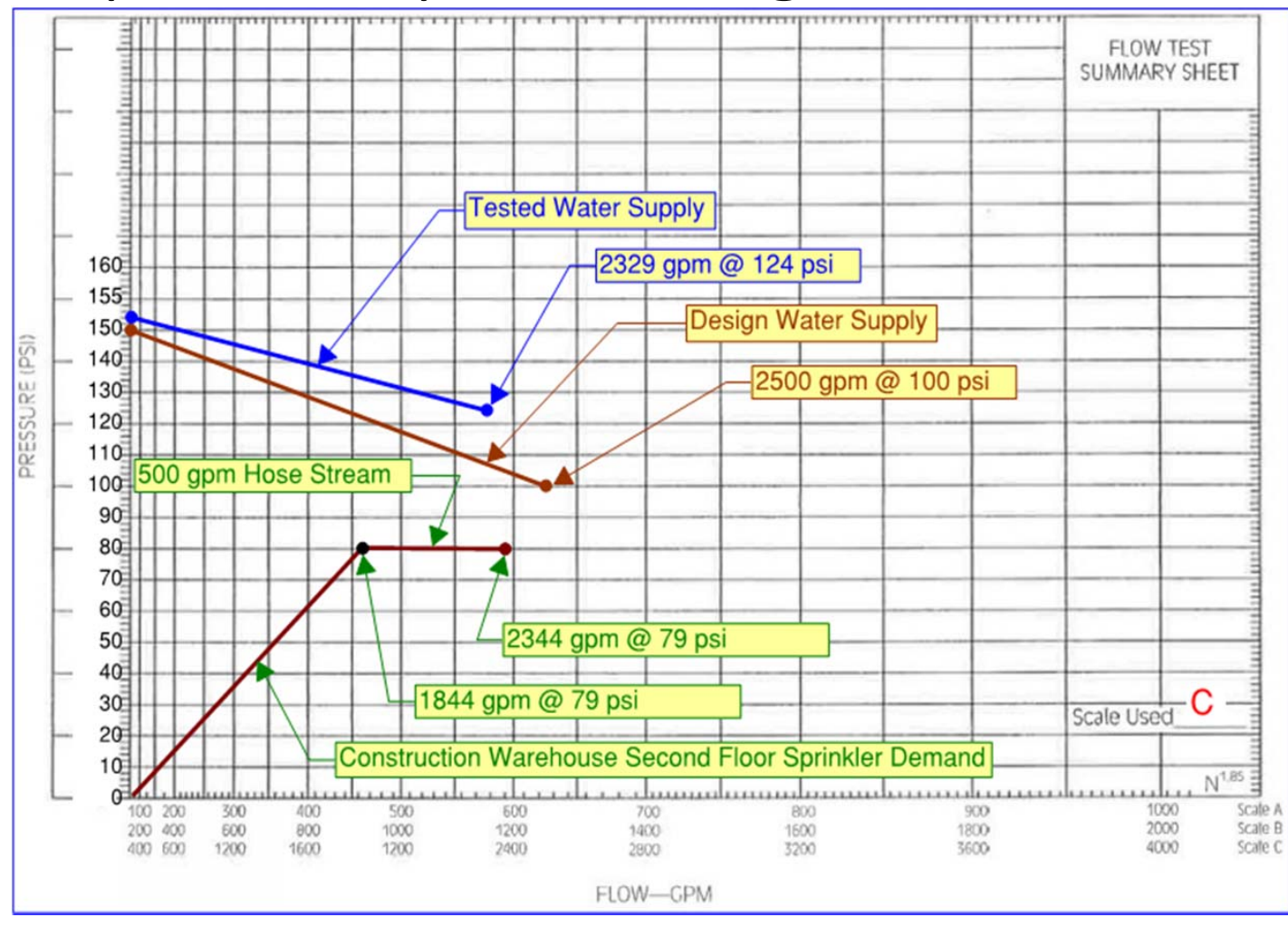




\section{Construction Warehouse - Fire Sprinkler System Design Criteria}

Main Floor Office \& Mezzanine, Loading Dock (Dry Pipe) \& Tool Crib Areas

- General

- Ordinary Hazard Group 2 Occupancy Classification

- Reliable Model G

- Standard Spray Upright (SSU) heads, $212^{\circ} \mathrm{F} \& 286^{\circ} \mathrm{F}, \mathrm{K}=5.6$

- Recessed Pendent (REC PEND) heads, $165^{\circ} \mathrm{F}, \mathrm{K}=5.6$

- Horizontal Sidewall (HSWL) heads, $212^{\circ} \mathrm{F}, \mathrm{K}=5.6$

- 500 gpm hose stream allowance 


\section{Construction Warehouse - Fire Alarm System}

- Building Fire Alarm System

- Serves the general fire protection needs of the Warehouse

- Provides for automatic notification of fire to building occupants

- Automatically shuts down supply ventilation on detection of smoke

- Monitors fire protection systems for alarm, supervisory and trouble signals

- Protected Premises Fire Alarm System

- Provides for automatic fire alarm notification to the Fire Department

- Automatically reports fire alarm, supervisory and trouble signals

- One-way High Frequency Radio transmitter 


\section{Construction Warehouse - Fire Alarm System}

- Main Fire Alarm Control Panel (FACP)

- Notifier ${ }^{\circledR}$ Model No. NFS2-3030

- Addressable

- Two (2) Signaling Line Circuits (SLCS)

- Located in Main Entry

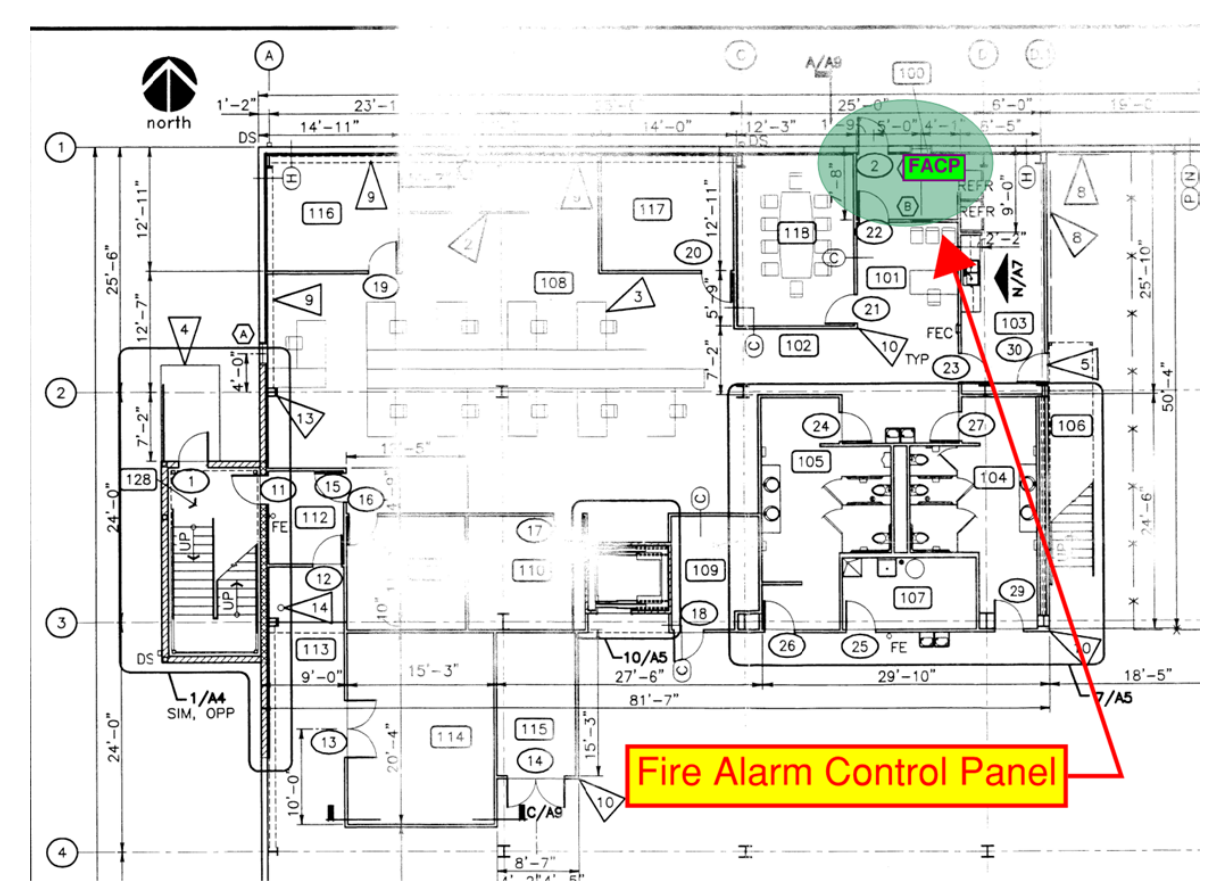




\section{Construction Warehouse - Fire Alarm System}

- Initiating Devices - All Initiate a Fire Alarm (Plus Other Functions)

- Manual Pull Stations at each exit on each floor

- Photoelectric Smoke Detectors (Elevator Recall Function)

- Near elevator door on every floor

- At the top of elevator hoistway

- In elevator machine room

- Photoelectric Duct Smoke Detectors (Shutdown AHU Supply Fans)

- Fixed Heat Detectors (Elevator Shunt Trip)

- In elevator machine room (w/i 24 inches of sprinkler head)

- At top of elevator hoistway (w/i 24 inches of sprinkler head) 


\section{Construction Warehouse - Fire Alarm System}

- Visible and Audible Notification Appliances

- Four Field Charger Power Supplies

- 13 Notification Appliance Circuits

- Visible and Audible Appliances provided

- Horn/Strobe Units

- Strobe Units

- Placement and location in accordance with NFPA 72

- Secondary Power Supplies

- Fire Alarm Control Panel - $26 \mathrm{~A}-\mathrm{H}$ battery (17.6 A-H required)

- Field Charger Power Supplies - $7 \mathrm{~A}-\mathrm{H}$ battery (1.9 A-H required) 


\section{Construction Warehouse Life Safety Performance Based Design}

- Objective

- Satisfy the following criteria:

Available Safe Egress Time (ASET) > Required Safe Egress Time (RSET) 


\section{Construction Warehouse Life Safety Performance Based Design}

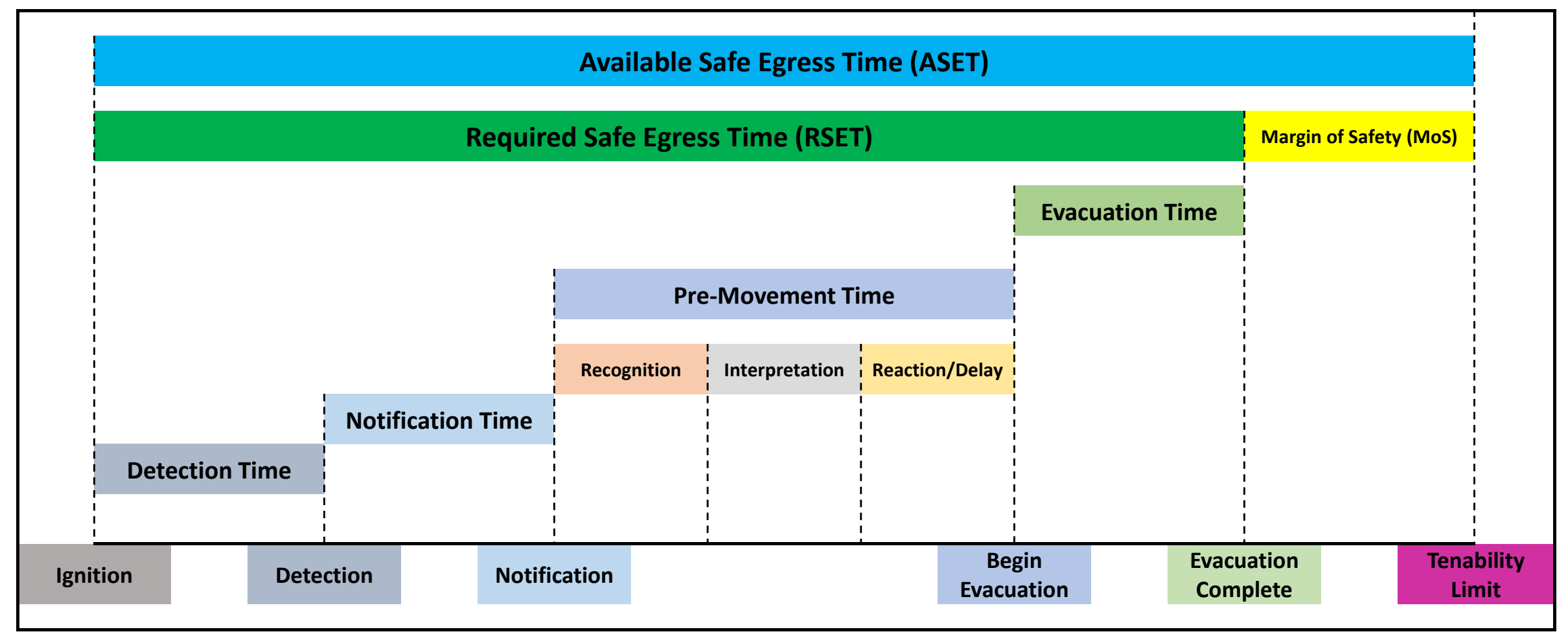


Construction Warehouse - Life Safety Performance Based Design Detection Time and Notification Time

- Detection Time

- Assessed by analysis of specific fire scenarios

- Hand calculations or computer fire model (FDS) results

- Detection assumed to occur upon the first

- Smoke detector alarm; or

- Sprinkler activation

- Notification Time

- Occupant notification is by Fire Alarm System notification appliances

- No time delay on sprinkler riser pressure switches

- Inherent time delay in processing fire alarm signals

- Notification Time: Assume 30 seconds 
Construction Warehouse - Life Safety Performance Based Design Pre-Movement Time

- Workers Are Trained

- Recognize and interpret fire notification signals

- Immediately evacuate in response to a fire alarm

- Visitors are escorted by a trained worker

- HOWEVER some workers are expected to delay movement

- Question fire alarm signal, wait until there is an indication of fire or smoke

- Investigate, seek out or warn others

- Collect personal items, shutdown computers and other equipment

- Attempt to help or rescue coworkers

- Attempt to put out a fire (e.g., with a fire extinguisher)

- Pre-Movement Time: Assume 60 seconds 


\section{Construction Warehouse - Life Safety Performance Based Design}

\section{Evacuation Time}

- Egress Analysis

- Hand Calculations and Pathfinder Software Egress Model

- Exterior Stairwells are 2-hour fire-resistive exit enclosures

- Evacuation time $\square$ all occupants pass into a protected stairwell enclosure

- West Stairwell is most limiting

\begin{tabular}{|c|c|c|c|}
\hline \multicolumn{4}{|c|}{ Time to Clear Each Floor and Number of Occupants (West Stairwell) } \\
\hline Floor Level & $\begin{array}{c}\text { Hand C alc } \\
\text { seconds } \\
\text { (\# occupants) }\end{array}$ & $\begin{array}{c}\text { Path finder (SFPE) } \\
\text { seconds } \\
\text { (\# occupants) }\end{array}$ & $\begin{array}{l}\text { Pathfind er (STEERING) } \\
\text { seconds } \\
\text { (H)ecupsuits) }\end{array}$ \\
\hline Second Floor & 159 seconds (103) & 176 seconds (86) & 158 seconds $(100)$ \\
\hline Office Mezzanine & 71 seconds (33) & 147 seconds (48) & 132 seconds $(40)$ \\
\hline Main Floor & Not Calculated (66) & 136 seconds (67) & 117 seconds (63) \\
\hline $\begin{array}{r}\text { TOTAL OCCUPANTS } \\
\text { USING WEST STAIRWELL }\end{array}$ & 202 & 201 & 209 \\
\hline
\end{tabular}

- Evacuation Time: 158 seconds 
Construction Warehouse - Life Safety Performance Based Design Life Safety Performance Criteria

- NFPA 101 (2012) Life Safety Code ${ }^{\circledR}$

5.2.2* Performance Criterion.

Any occupant who is not intimate with ignition shall not be exposed to instantaneous or cumulative untenable conditions. 


\section{Construction Warehouse - Life Safety Performance Based Design Tenability Limits}

- Tenability limits established to limit

- Sensory irritation and visual impairment (VISIBILITY)

- Thermal effects to building occupants (TEMPERATURE)

- Depression of the central nervous system (CARBON MONOXIDE DOSE)

- Tenability criteria is measured at a

\begin{tabular}{|l|l|}
\hline \multicolumn{2}{|c|}{ Tenability Criteria } \\
\hline \multicolumn{1}{|c|}{ Parameter } & \multicolumn{1}{c|}{ Limit } \\
\hline Visibility & $\begin{array}{l}10 \mathrm{~m} \text { in Large Open Areas } \\
3 \mathrm{~m} \text { in Office Areas }\end{array}$ \\
\hline $\begin{array}{l}\text { Carbon Monoxide } \\
\text { Dose }\end{array}$ & 0.3 (FED) $[10,500 \mathrm{ppm}-\mathrm{min}]$ \\
\hline Temperature & $100^{\circ} \mathrm{C}$ \\
\hline
\end{tabular}
level $6 \mathrm{ft}$. $(1.82 \mathrm{~m})$ above the floor 


\section{Construction Warehouse - Fire Scenario Main Floor Storage Rack Fire}

- Rack Storage Fuel Array

- Cartons of Unexpanded Polystyrene Cups

- $1 \mathrm{~m} \times 1 \mathrm{~m} \times 1 \mathrm{~m}$ cartons

- $4 \times 4 \times 4$ carton array

- Each layer of 4 cartons stored on pallets in an open rack

- 18 ft. 6 in. ft. height / 24 ft. ceiling

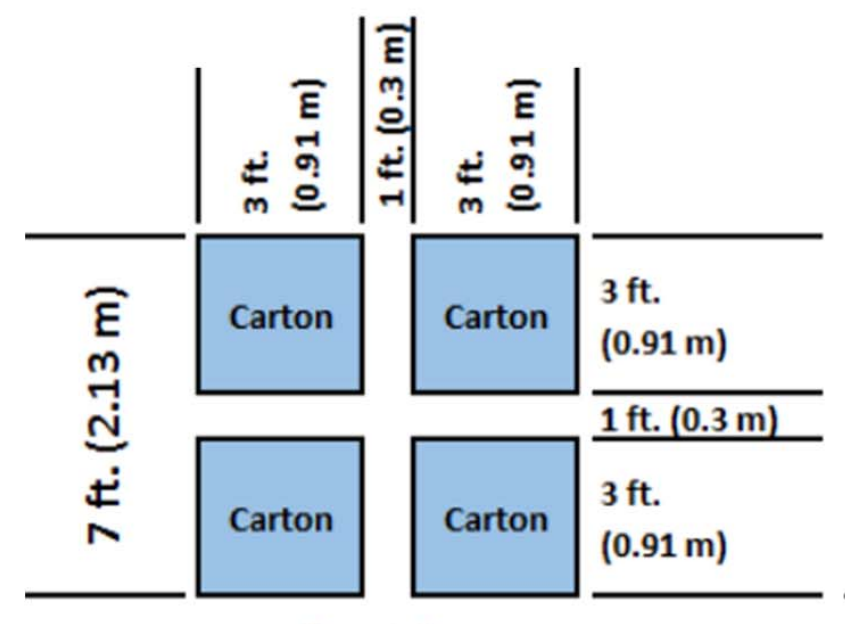

Plan View

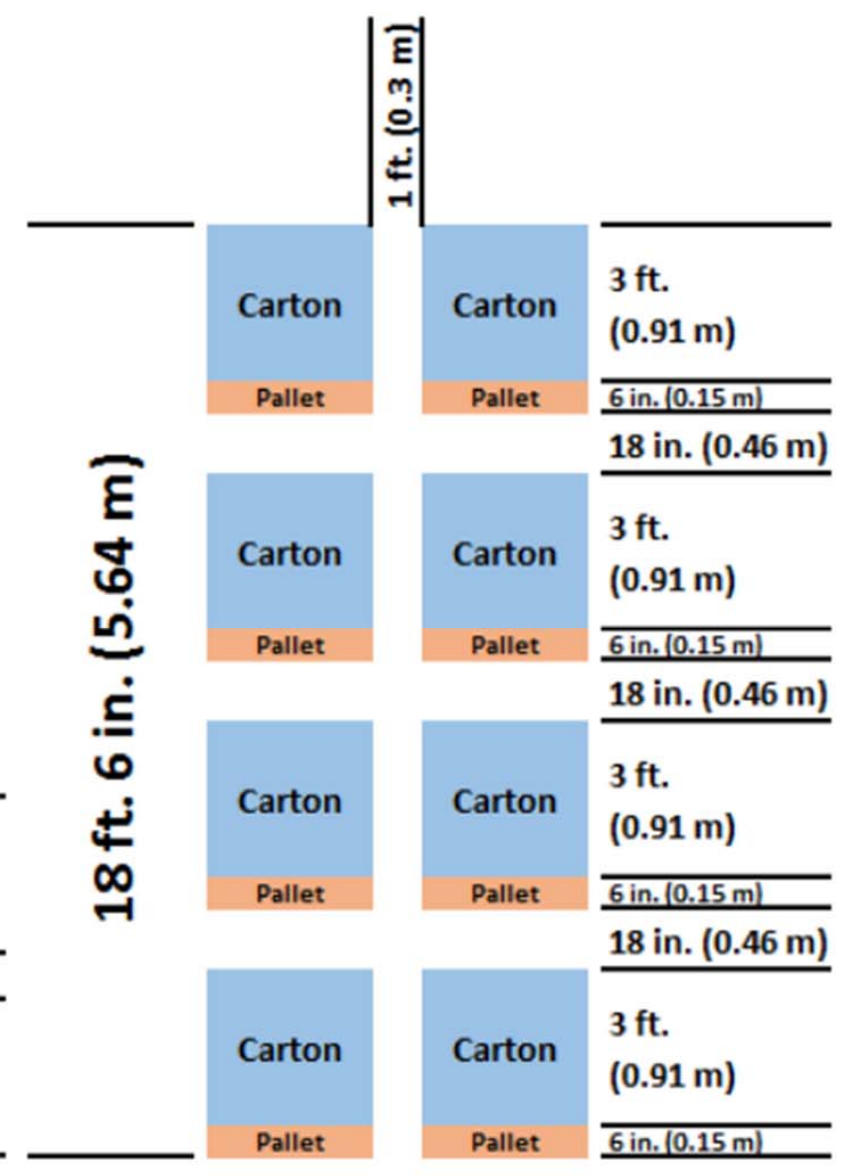

Elevation View 


\section{Construction Warehouse - Fire Scenario}

\section{Main Floor Storage Rack Fire}

- FDS Model

- Sixteen (16) $1 \mathrm{~m}^{2}$ Burners - Simulated Fuel Array

- Burners $1.3 \mathrm{~m}, 2.8 \mathrm{~m}, 4.3 \mathrm{~m}$ and $5.8 \mathrm{~m}$ (Top) above the floor

- Peak Heat Release Rate

- "t-cubed" growth / ramp controlled in FDS input

- Sequenced ignition - 0, 30, 60, 90 seconds

- 36,500 kW @ 150 seconds (If not controlled by sprinklers)

- $Y_{\mathrm{S}}=0.074 \mathrm{~g} / \mathrm{g} ; \mathrm{Y}_{\mathrm{CO}}=0.026 \mathrm{~g} / \mathrm{g}$

- Mass Weighted Average $Y_{S}$ and $Y_{C O}$ (SFPE Hdbk, $3^{\text {rd }}$ Edition, Table 3-4.14)

- Corrugated Cardboard and Unexpanded Polystyrene

- Basis

- Heskestad Analyses of Rack Storage Tests (SFPE Hdbk, $3^{\text {rd }}$ Edition, Chapter 3-1, "Heat Release Rates")

Table 3-1.5 HRR Values of Palletized and Rack Storage Commodities Tested at FMRC

\begin{tabular}{llcccc}
\hline Test & Commodity & $\begin{array}{c}\text { Storage } \\
\text { Height } \\
(\mathrm{m})\end{array}$ & $\begin{array}{c}\text { Peak } \\
\text { HRR } \\
\left(\mathrm{kW} \cdot \mathrm{m}^{-2}\right)\end{array}$ & $\begin{array}{c}\text { Time of } \\
\text { Peak } \\
(\mathrm{s})\end{array}$ \\
\hline RS-7 & PS cups in compartmented CB cartons & 2.90 & 4,420 & 95 \\
RS-8 & $\cdots$ & 2.90 & 4,420 & 100 \\
RS-9 & $\cdots$ & 2.90 & 4,420 & 120 \\
RS-10 & $\cdots$ & 4.42 & 6.580 & 100 \\
\hline RS-11 & $\cdots$ & 5.94 & 8,030 & 148 \\
\hline
\end{tabular}




\section{Construction Warehouse - Fire Scenario Main Floor Storage Rack Fire - Devices}

- HVAC

- (2) $17,500 \mathrm{cfm}$ exhaust fans normally "OFF", "ON" at $90^{\circ} \mathrm{F}$

- (1) 1,750 cfm exhaust fan always "ON"

- (4) Open wall vents

- Devices

- Ceiling temperature detectors $24 \mathrm{ft}$. above the floor

- (1) On ceiling at center of fuel array

- (4) On ceiling equally spaced $4.3 \mathrm{~m}$ (10 ft.) from centerline

- Radiative Flux detectors

- (8) Trees -6 detectors spaced $1.2 \mathrm{~m}$ (3.9 ft.) vertically

- Located $2.4 \mathrm{~m}$ (8 ft.) and $4.8 \mathrm{~m}$ (16 ft.) from each edge of fuel array

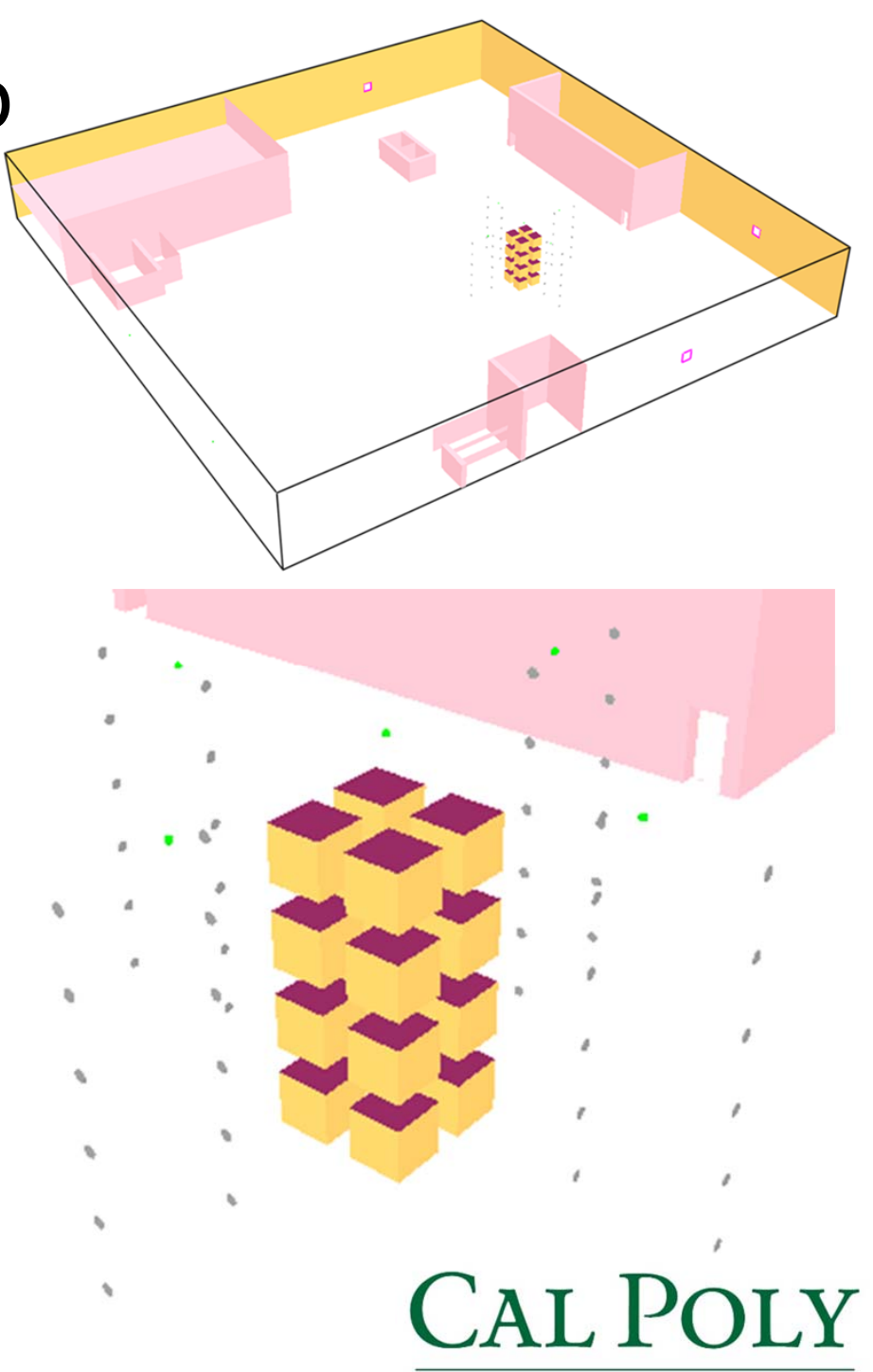

Fire Protection Engineering 


\section{Construction Warehouse - Fi
Main Floor Storage Rack Fire}

- Two cases evaluated for this scenario

- Case 1 - Sprinklers permitted

- DETACT

- Sprinkler activation at 60 seconds

- Fire controlled at $2,592 \mathrm{~kW}$

- Tenability analysis

- Evaluate potential for fire spread to adjacent commodities

- Case 2 - Sprinklers not permitted

- Growth to 36,500 kW @ 150 seconds

- Evaluate potential for fire spread to adjacent commodities

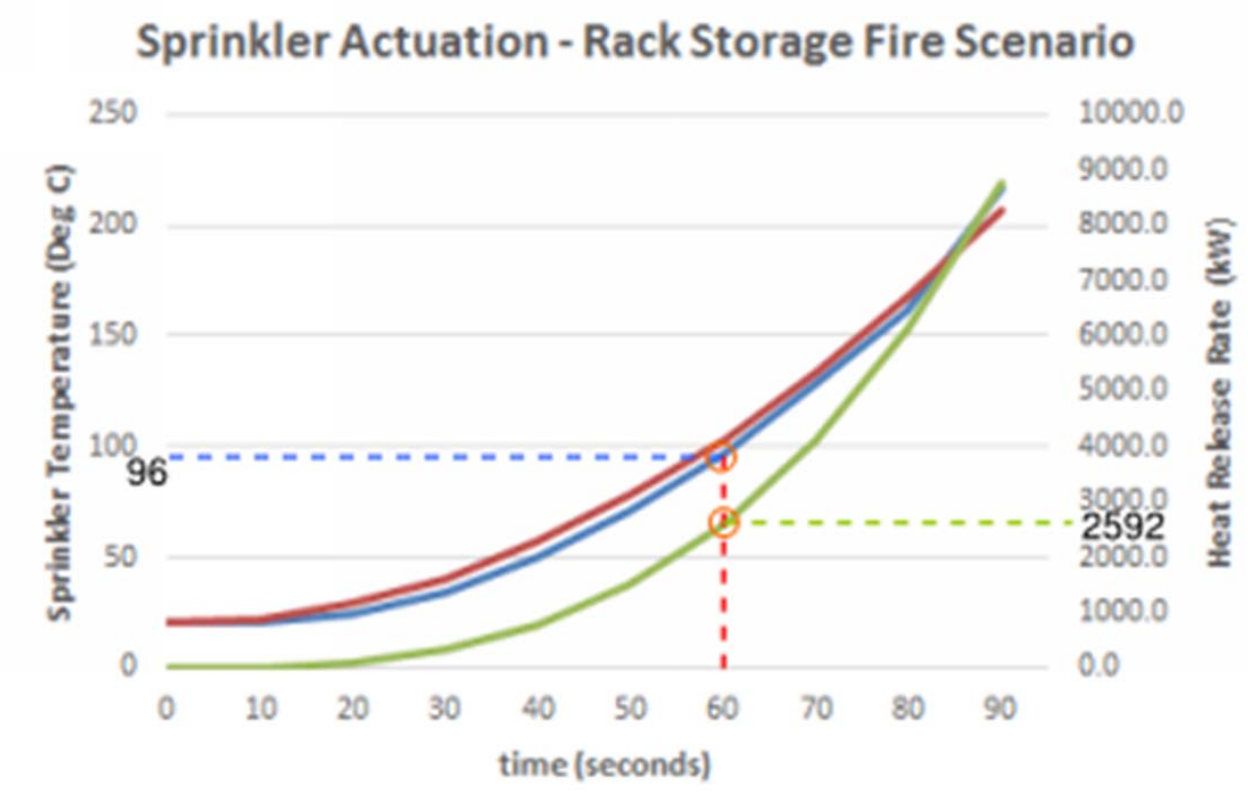

-Sprinkler Temp — Gas Temp W Heat Release Rate 


\section{Construction Warehouse - Fire Scenario Main Floor Storage Rack Fire}

- Model Results

- Case 1 - Sprinklers permitted

- Assess fire dynamics issues:

- Max. temperature at ceiling in center of plume $180^{\circ} \mathrm{C}$ $\left(356^{\circ} \mathrm{F}\right) @ 60$ seconds

Rack Storage Fire Ceiling Temperature - Sprinklers

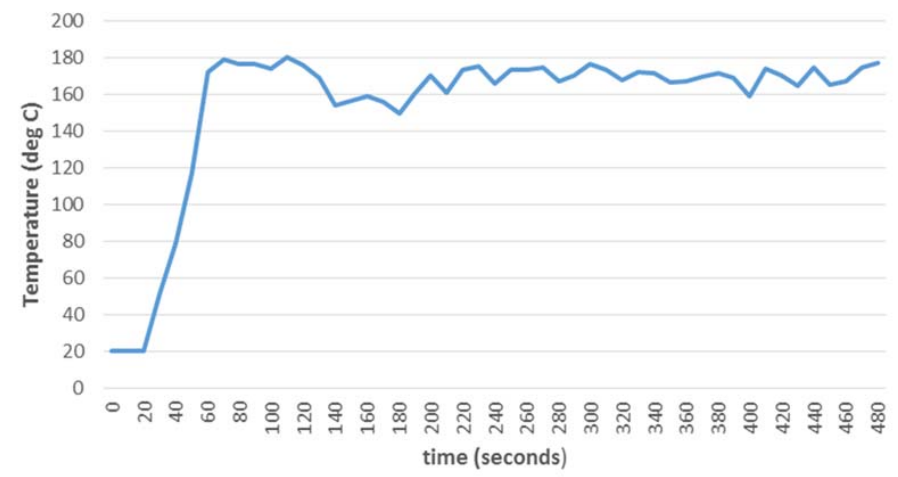

- Rad flux rises to approximately $4.0 \mathrm{~kW} / \mathrm{m}^{2} @ 8 \mathrm{ft}$. and 1.5 kW/m²@16 ft. @ 180 seconds

- Critical Heat Flux (CHF) is $10 \mathrm{~kW} / \mathrm{m}^{2}$ for corrugated cardboard

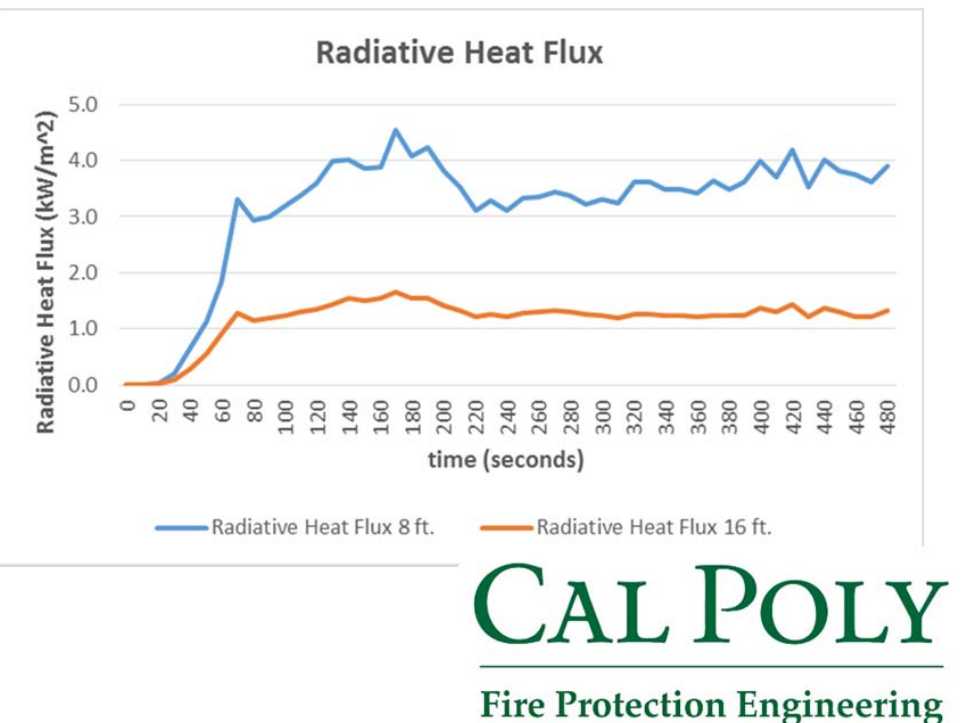




\section{Construction Warehouse - Fire Scenario}

Main Floor Storage Rack Fire

- Model Results

- Case 1 - Sprinklers permitted

- Tenability

- Visibility Criteria

- Exceeds $10 \mathrm{~m}$ limit

- Time $=400$ seconds

- $1.82 \mathrm{~m}$ above floor

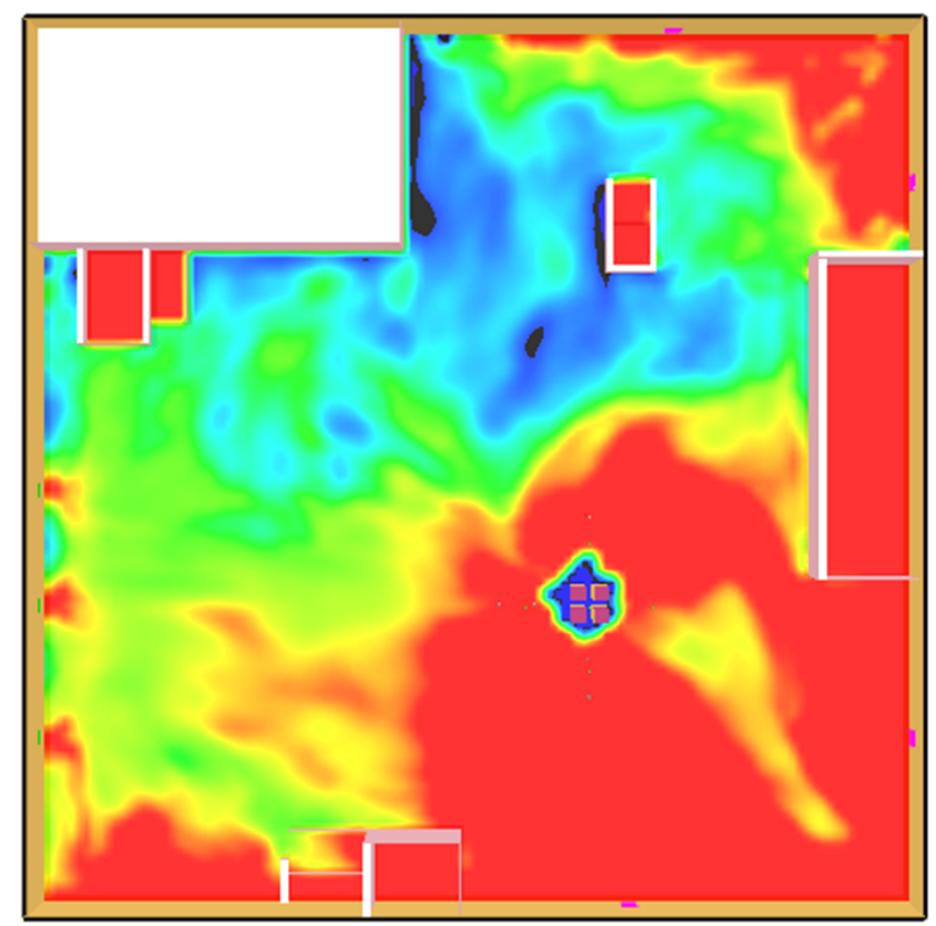




\section{Construction Warehouse - Fire Scenario}

Main Floor Storage Rack Fire

- Model Results

- Case 1 - Sprinklers permitted

- Tenability

- Temperature Criteria

- $100^{\circ} \mathrm{C}$ not exceeded $\left(60^{\circ} \mathrm{C}\right.$ not exceeded)

- Time $=480$ seconds

- $1.82 \mathrm{~m}$ above floor

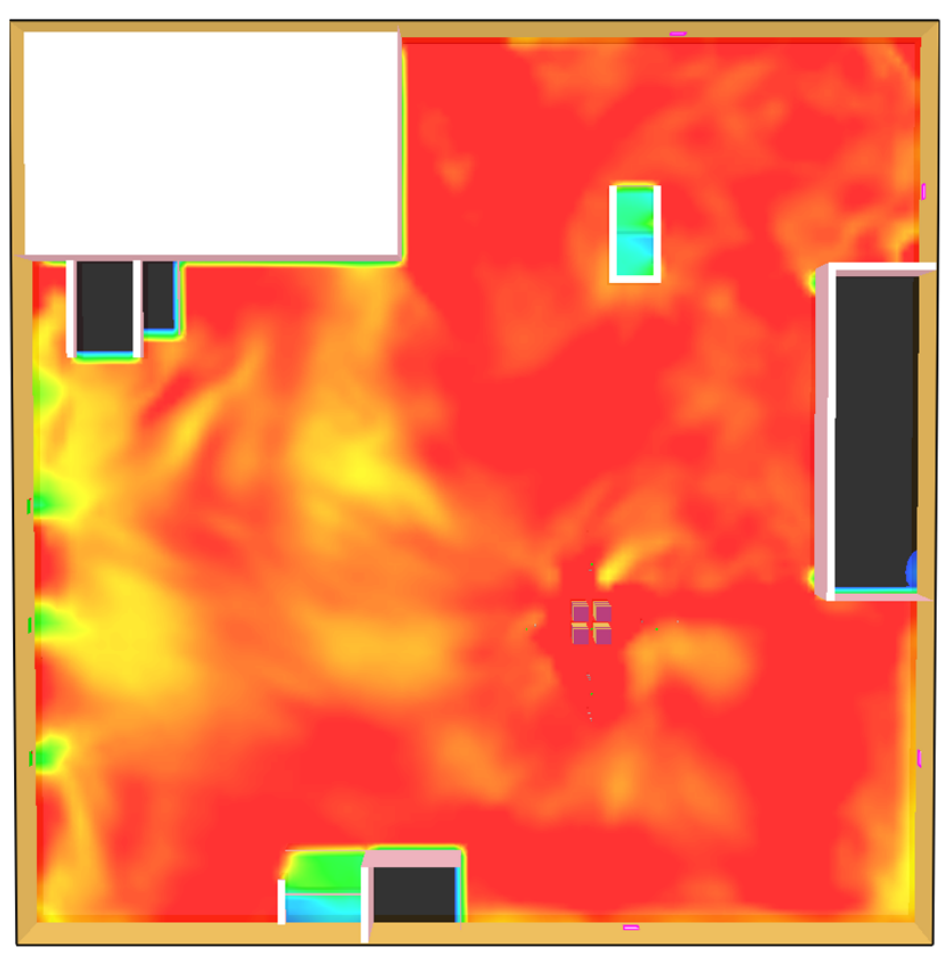




\section{Construction Warehouse - Fire Scenario Main Floor Storage Rack Fire}

- Model Results

- Case 1 - Sprinklers permitted

- Tenability

- Carbon Monoxide Dose

- 100 ppm-min (FED = 0.003)

- Time $=480$ seconds

- FED of 0.3 is not exceeded

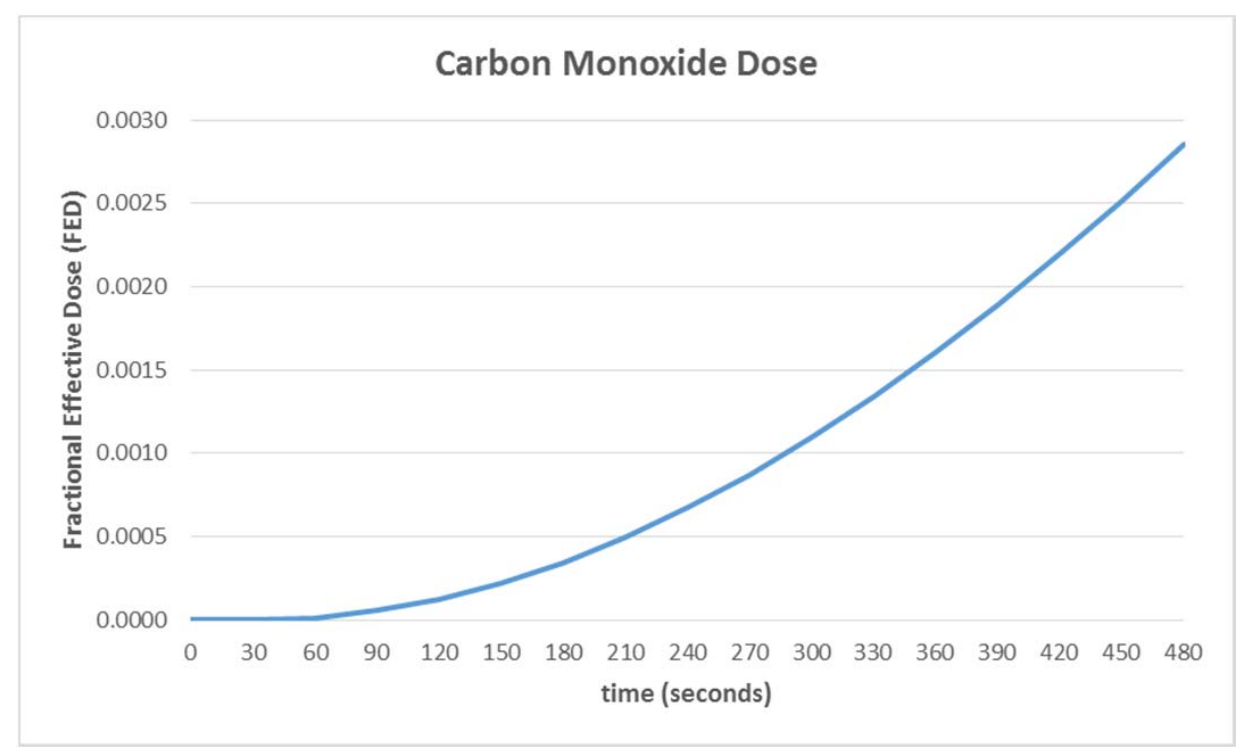




\section{Construction Warehouse - Fire Scenario Main Floor Storage Rack Fire}

- Model Results

- Case 1 -Sprinklers permitted

- Assess ASET vs. RSET

- $\mathrm{ASET}=400$ seconds (based on visibility)

- RSET

- $\mathrm{RSET}=60+30+60+158=\underline{308 \text { seconds }}$

- RSET (308 sec) < ASET (400 sec) $\rightarrow$ GOOD!!

- Margin of Safety

- $\mathrm{MoS}=400-308=92$ seconds $\rightarrow 30 \%$ $t_{\text {detection }}=60$ seconds

$t_{\text {notification }}=30$ seconds

$\mathrm{t}_{\text {pre-movement }}=60$ seconds

$t_{\text {evacuation }}=158$ seconds 


\section{Construction Warehouse - Fire Scenario}

\section{Main Floor Storage Rack Fire}

- Model Results

- Case 2 - Sprinklers Not Permitted

- Assess fire dynamics issues:

- Max. temperature at ceiling in center of plume $877^{\circ} \mathrm{C}$ $\left(1,611^{\circ} \mathrm{F}\right) @ 130$ seconds

- $538^{\circ} \mathrm{C}\left(1,000^{\circ} \mathrm{F}\right)$ is exceeded

- Ceiling / second floor damage is likely

- Rad flux trends (erratic)

- Trends to $50 \mathrm{~kW} / \mathrm{m}^{2}$ over 480 seconds @ $8 \mathrm{ft}$.

- Trends to $10 \mathrm{~kW} / \mathrm{m}^{2}$ over 480 seconds @ $16 \mathrm{ft}$.

- Critical Heat Flux (CHF) is $10 \mathrm{~kW} / \mathrm{m}^{2}$ for corrugated cardboard

- $\mathrm{CHF}$ is exceeded - fire spread to adjacent commodities is predicted

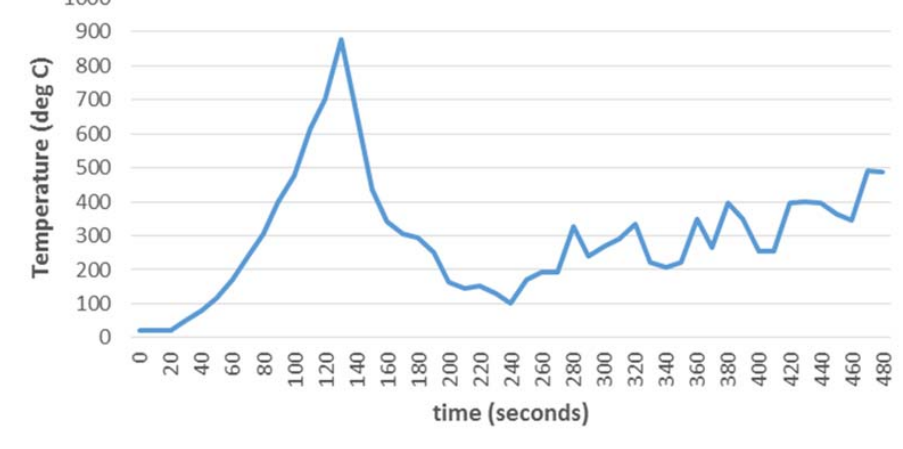

Ceiling Temperature - No Sprinkler Activation

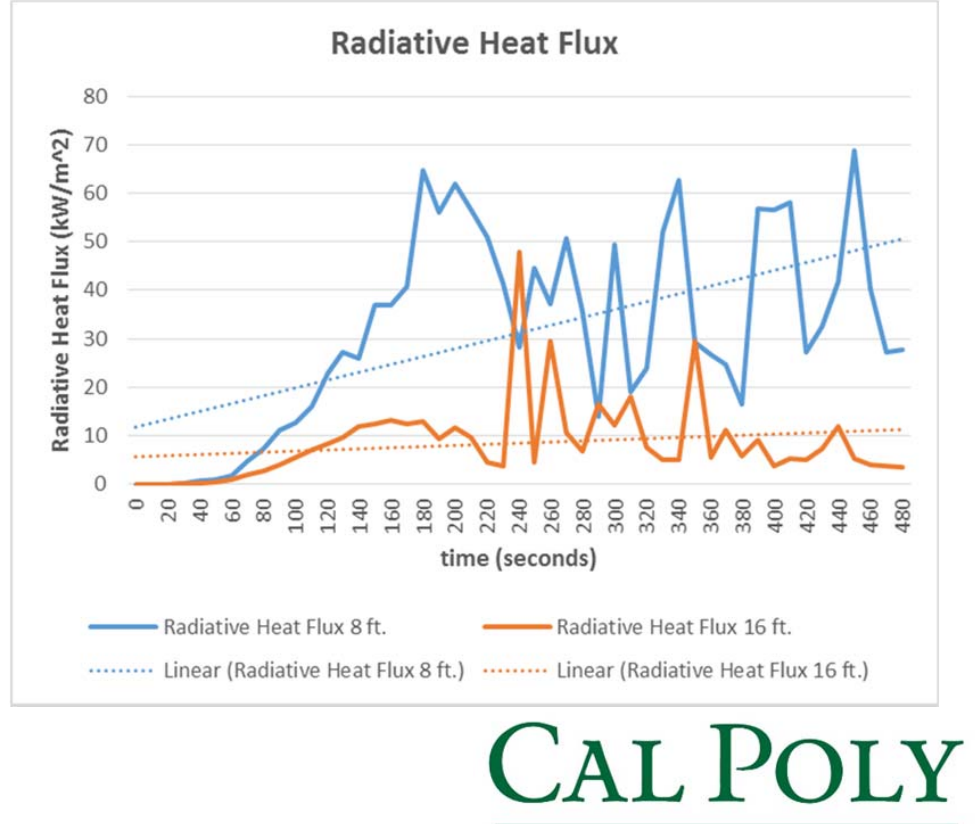

Fire Protection Engineering 


\section{Construction Warehouse - Fire Scenario Main Floor Storage Rack Fire}

- Model Results

- Conclusions

- Life Safety performance based design criteria is met

- Fire sprinklers are essential to survivability of the construction warehouse

- Without sprinklers

- Rapid fire growth

- An uncontrollable fire would result

- Danger to building occupants and hampered safe egress

- Spread of fire to adjacent commodities is predicted

- Ceiling temperature would cause significant damage to the second floor 


\section{Construction Warehouse - Fire Scenario Mezzanine Office Area (Workstation) Fire}

- FDS Model

- Standard $8 \mathrm{ft}$. $x 8 \mathrm{ft}$. Office Cubicle Fire

- $1 \mathrm{~m}^{2}$ Burner / 32 inches above floor

- Peak Heat Release Rate

- "t-squared" growth

- 6,730 kW @ 530 seconds

- $Y_{\mathrm{S}}=0.037 \mathrm{~g} / \mathrm{g} ; \mathrm{Y}_{\mathrm{CO}}=0.022 \mathrm{~g} / \mathrm{g}$

- Mass Weighted Average $Y_{S}$ and $Y_{C O}$

(SFPE Hdbk, $3^{\text {rd }}$ Edition, Table 3-4.14)

- Wood, expanded and unexpanded plastic, ABS, Nylon

- Basis

- NIST NCSTAR 1-5C, Federal Building and Fire Safety Investigation of the World Trade Center Disaster, "Fire Tests of Single Office Workstations"

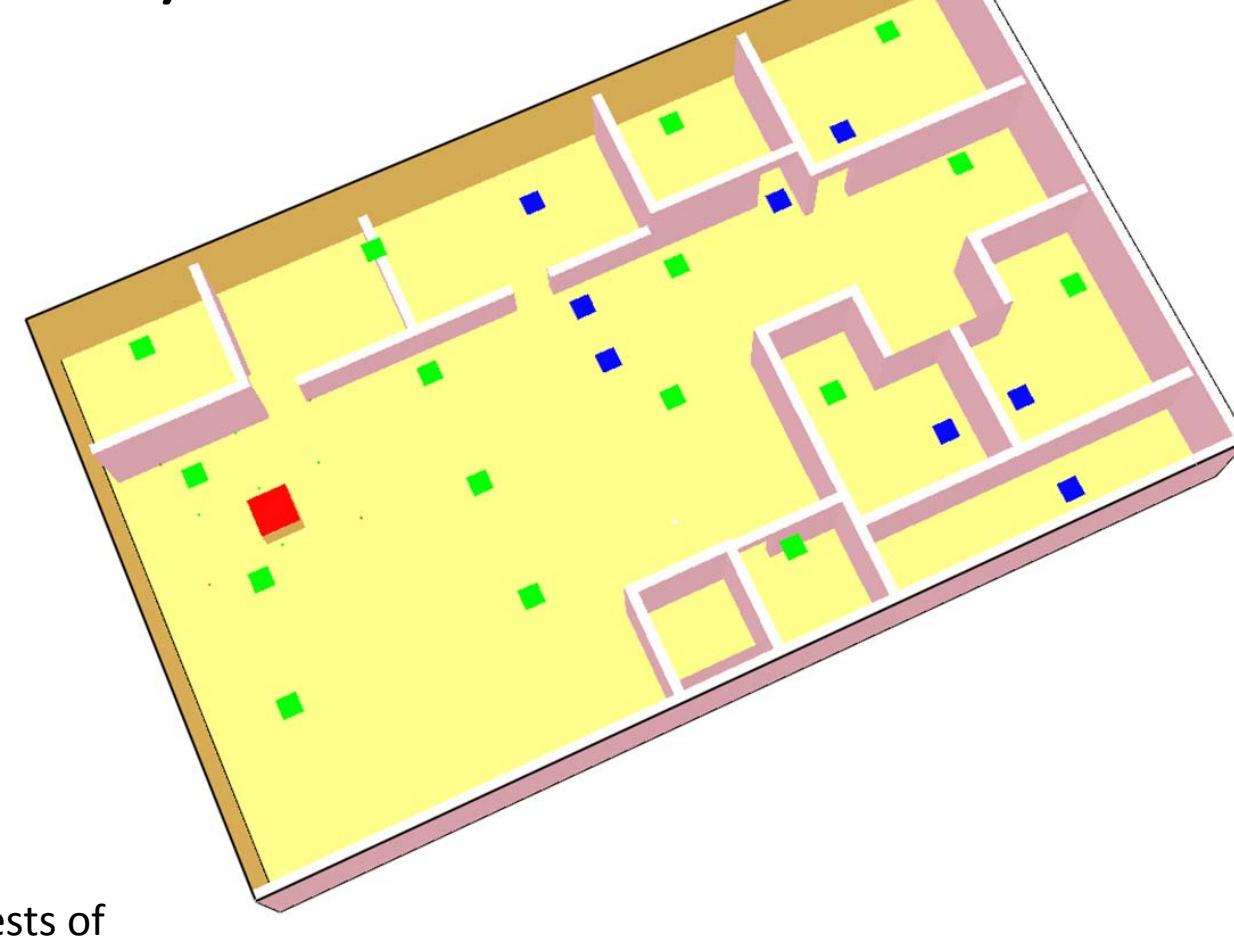




\section{Construction Warehouse - Fire Scenario}

Mezzanine Office Area (Workstation) Fire - Devices

- HVAC

- $400 \mathrm{cfm}$ exhaust fan

- 2,700 cfm return air

- 3,100 cfm supply air

- Devices

- Ceiling Smoke Detector

- (1) Elevator Lobby 


\section{Construction Warehouse - Fire Scenario Mezzanine Office Area (Workstation) Fire}

- Sprinkler Activation

- DETACT model - 170 seconds

- Fire controlled at $694 \mathrm{~kW}$

- Smoke Detector Activation

- Hand calculation - 54 seconds

- FDS - 62 seconds

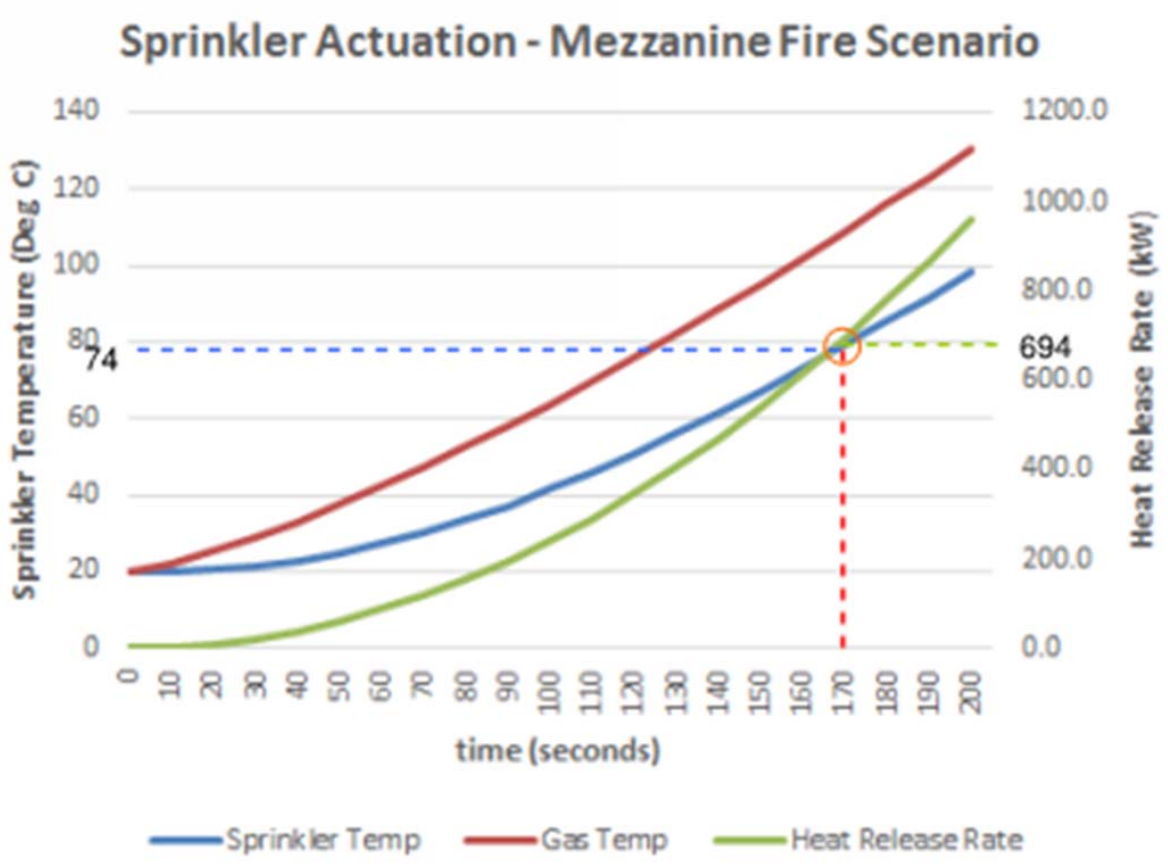




\section{Construction Warehouse - Fire Scenario}

Mezzanine Office Area (Workstation) Fire

- Model Results

- Tenability

- Visibility Criteria

- Exceeds $3 \mathrm{~m}$ limit

- Time $=130$ seconds

- $1.82 \mathrm{~m}$ above floor

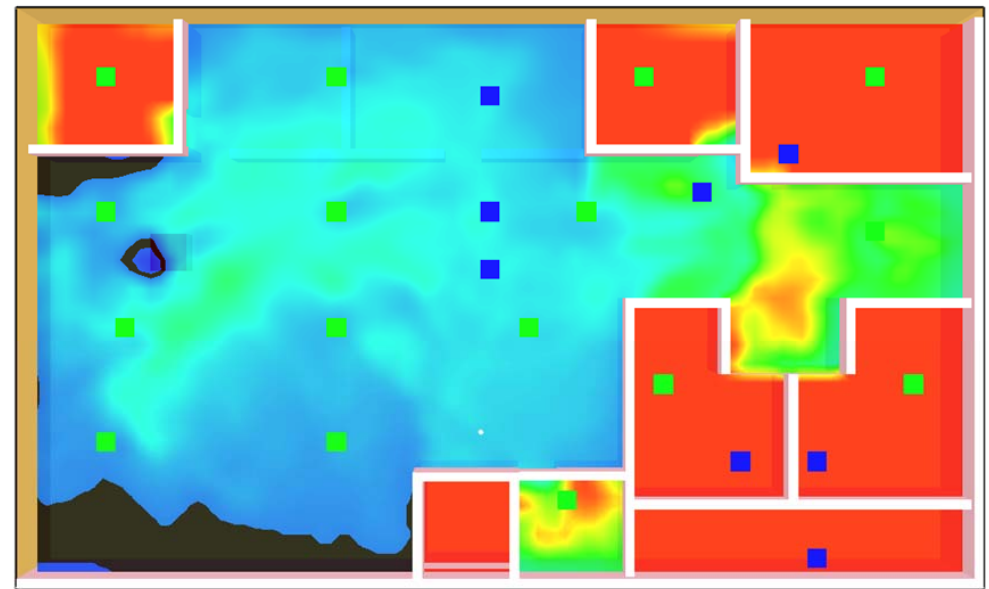




\section{Construction Warehouse - Fire Scenario Mezzanine Office Area (Workstation) Fire}

- Model Results

- Tenability

- Temperature Criteria

- $100^{\circ} \mathrm{C}$ exceeded

- Time $=140$ seconds

- $1.82 \mathrm{~m}$ above floor

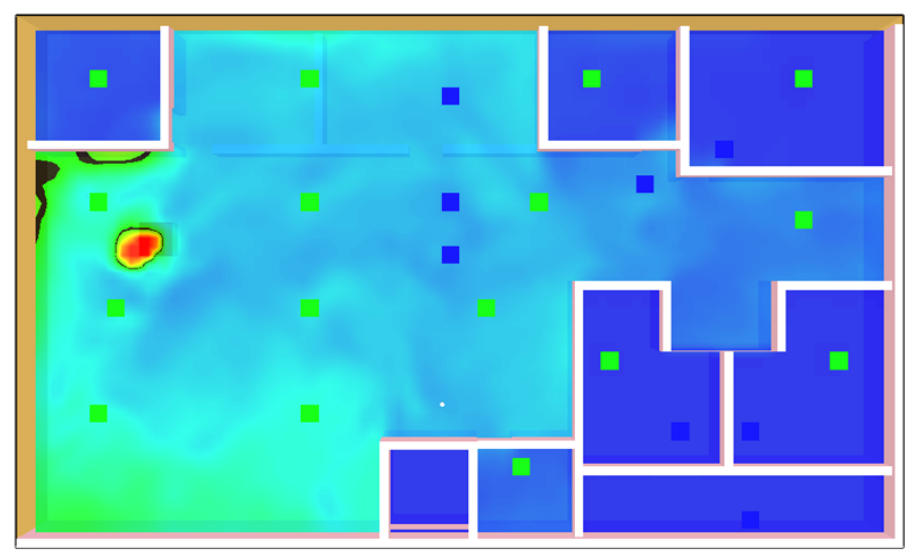




\section{Construction Warehouse - Fire Scenario Mezzanine Office Area (Workstation) Fire}

- Model Results

- Tenability

- Carbon Monoxide Dose

- 468 ppm-min (FED = 0.013)

- Time $=480$ seconds

FED of 0.3 is not exceeded

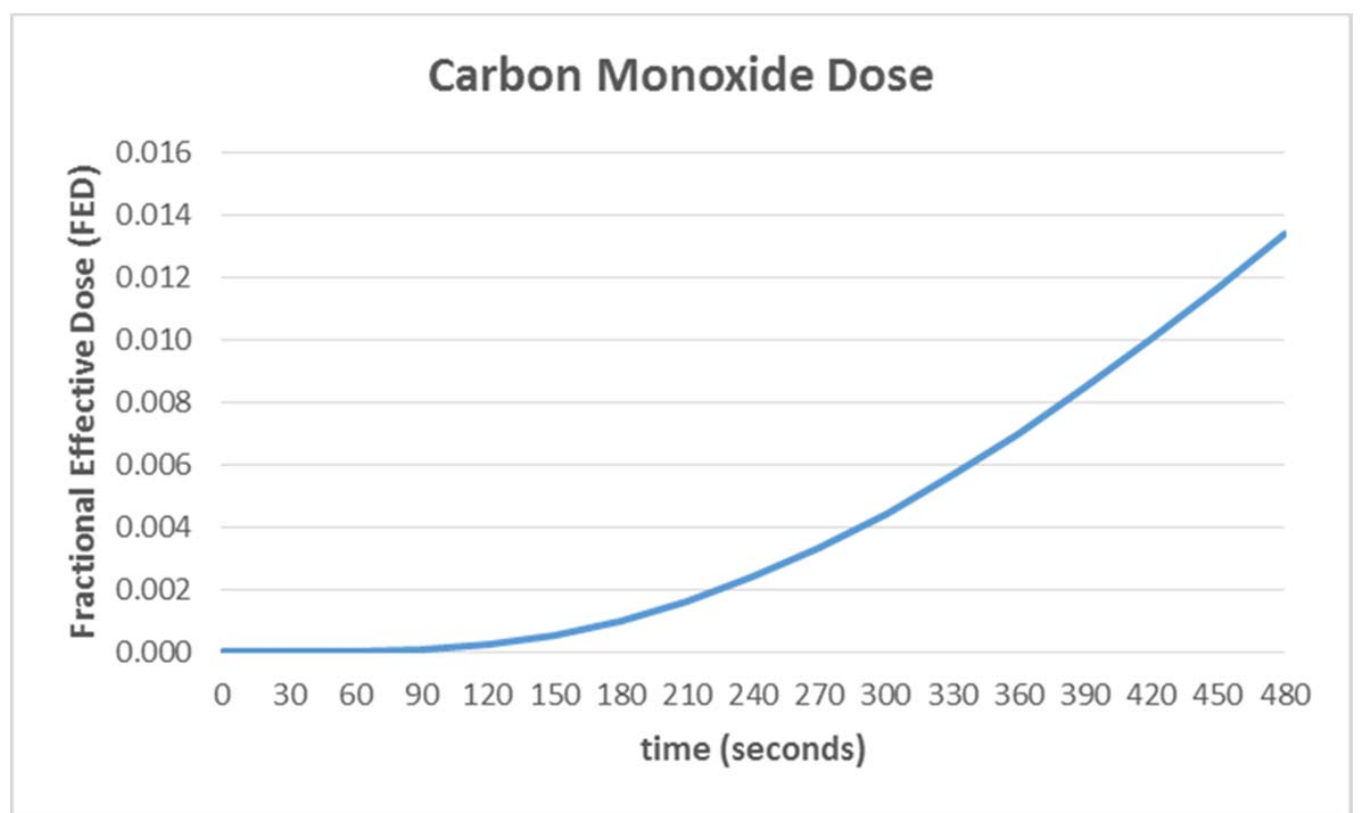




\section{Construction Warehouse - Fire Scenario Mezzanine Office Area (Workstation) Fire}

- Assess ASET vs. RSET

$t_{\text {detection }}=54$ seconds

$t_{\text {notification }}=30$ seconds

- ASET = 130 seconds (based on visibility)

- RSET

$t_{\text {pre-movement }}=60$ seconds

$t_{\text {evacuation }}=158$ seconds

- $\mathrm{RSET}=54+30+60+158=\underline{302 \text { seconds }}$

- RSET (302 sec) > ASET (130 sec) $\rightarrow$ NO GOOD!!

NOTE: Deficit $($ RSET - ASET $)=172$ seconds 


\section{Construction Warehouse - Fire Scenario Mezzanine Office Area (Workstation) Fire}

- Alternatives - (Must make up 172 second deficit)

1. Replace standard response sprinklers with quick response sprinklers

- DETACT - Improves sprinkler response by 30 seconds / RSET reduction of 30 seconds

2. Remove suspended ceiling / replace sprinklers with quick response sprinklers

- DETACT + FDS - Increases visibility tenability limit (ASET) by 80 seconds

3. Add a protected exit to mezzanine office area on north exterior wall

- Pathfinder - Improves time for all building occupants to exit / RSET reduction of 42 seconds

4. Install mechanical smoke control system

- FDS - Increases visibility tenability limit (ASET) indefinitely

5. Remove mezzanine walls - open to main floor storage area

- Rack Fire Scenario becomes the bounding scenario / MoS = 92 seconds

- Impact of Rack Fire Scenario on mezzanine occupants must be considered

6. Do nothing / control number of occupants / control combustible loading

- Meets prescriptive requirement

- Refine performance based design analysis 
Thank-You!

CAL POLY

Fire Protection Engineering 